\title{
A scaling approach to the derivation of hydrodynamic boundary conditions
}

\author{
TIEZHENG QIAN ${ }^{1}$, CHUNYIN QIU ${ }^{1}$ AND PING SHENG ${ }^{2}$ \\ ${ }^{1}$ Department of Mathematics, The Hong Kong University of Science and Technology, Clear Water Bay, \\ Kowloon, Hong Kong, China \\ ${ }^{2}$ Department of Physics and Institute of Nano Science and Technology, The Hong Kong University of \\ Science and Technology, Clear Water Bay, Kowloon, Hong Kong, China
}

(Received 14 September 2007 and in revised form 1 June 2008)

We show hydrodynamic boundary conditions to be the inherent consequence of the Onsager principle of minimum energy dissipation, provided the relevant effects of the wall potential appear within a thin fluid layer next to the solid wall, denoted the surface layer. The condition that the effect of the surface layer on the bulk hydrodynamics must be independent of its thickness $h$ is shown to imply a set of consistent 'scaling relationships' between $h$ and the surface-layer variables/parameters. The use of the scaling relations, in conjunction with the surface-layer equations of motion derived from the Onsager principle, directly leads to the hydrodynamic boundary conditions. We demonstrate the surface-layer scaling process both physically and mathematically, and relate the parameters of the boundary conditions to those in the surface-layer equations of motion. In spatial regions outside the surface layer, equivalence between the use of surface-layer dynamics and boundary conditions is numerically demonstrated for Couette flows. As an application of the present approach, we derive the liquid-crystal hydrodynamic boundary conditions in which the rotational and translational dynamics are coupled.

\section{Introduction}

Boundary conditions complement the equations of motion and constitutive equations as a key element of continuum hydrodynamics. In spite of their importance, however, not all boundary conditions have been derived from basic principles. A prominent example is the no-slip boundary condition (NSBC) (Batchelor 1967), which has been widely employed but recognized some time ago to be incompatible with immiscible flows that involve a moving contact line, defined to be the intersection of the fluid-fluid interface with the solid wall (Moffatt 1964; Huh \& Scriven 1971; Dussan V. \& Davis 1974; Dussan V. 1976, 1979; de Gennes 1985; Koplik, Banavar \& Willemsen 1988; Thompson \& Robbins 1989). Recently, it was discovered that the use of Onsager principle of minimum energy dissipation (Onsager 1931a, b) can yield the required boundary conditions, denoted the generalized Navier boundary conditions (GNBCs), for resolving the moving contact line problem (Qian, Wang \& Sheng 2003, 2004, 2006). This success suggests an approach, based on the Onsager principle, for the derivation of hydrodynamic boundary conditions.

In this work we present a systematic scheme for deriving the hydrodynamic boundary conditions that is based on two elements. The first is the physical concept 
of a surface layer, arising from the consideration that the (solid) wall potential is the source of the boundary conditions, but the wall potential and its influence have a small but finite extent $h$. That is, within the surface layer, the effect of the wall on the fluid grows from zero, at the zero position usually associated with the (centre of mass) location of the solid wall molecules, to saturation at some finite distance $h$ into the fluid. The introduction of the surface layer immediately implies the second element of the scheme: the application of the Onsager principle to all the dissipations that occur beyond zero but within the surface layer, leading to a set of equations of motion that may be derived in the same manner as in the bulk. The boundary conditions to be applied at zero are natural and straightforward: zero viscous stress and zero Young stress as there is no fluid beyond zero.

We show that the continuum hydrodynamic boundary conditions emerge, through a set of scaling relations, as the leading-order terms of the equations of motion in the surface layer, that are independent of the surface-layer thickness $h$ as $h \rightarrow 0^{+}-$ i.e. the continuum boundary conditions preserve the leading-order hydrodynamics in the limit of $h \rightarrow 0^{+}$, and hence the hydrodynamic solution in the bulk. This is the desired result because the boundary conditions describe the integrated effects of the surface layer and hence thickness independence is expected in the limit of $h \rightarrow 0^{+}$. Besides putting the linkage between the hydrodynamic boundary conditions and the Onsager principle on a better physical and mathematical basis than previously attained, another benefit of the scaling approach is the demonstration that the Allen-Cahn relaxational boundary condition in the GNBC (Qian et al. 2003, 2006) is a direct consequence of diffusive dynamics in the surface layer, and there is a direct relationship between the surface-layer mobility coefficient $M$ and the AllenCahn relaxational rate parameter $\Gamma$. In addition, the scaling approach also allows easy generalization to the derivation of hydrodynamic boundary conditions in more complex systems, e.g. liquid crystals. In what follows, the formulation of the approach is presented in $\S 2$, followed by the description of scaling relations for obtaining the continuum boundary conditions in $\S 3$. The near-equivalence of surface layer and boundary conditions, in terms of the hydrodynamic solutions, is numerically demonstrated in $\S 4$. In $\S 5$ we illustrate the present approach by deriving the boundary conditions for liquid-crystal hydrodynamics, where the presence of both rotational and translational motions implies non-trivial coupling between the two. In $\S 6$ we conclude by comparing with the kinetic theory approach and noting some implications.

\section{Formulation}

\subsection{Onsager principle of minimum energy dissipation}

Since the early work of Helmholtz, there has been a steady development of variational principles involving dissipation (Rayleigh 1873). Here we attribute to Onsager the version that foreshadowed many later developments in statistical mechanics of dissipative systems. The Onsager principle concerns the rules governing the irreversible dissipative processes in the regime of linear response. According to this principle, the variational minimization of a functional would yield the equations of motion that govern the most probable course of a dissipative process (Onsager 1931a,b). In the statistical sense, the most probable course is the only course of action observed macroscopically. For completeness, we present below an outline of the Onsager principle. 
For the general case of multiple dynamic variables $q_{1}, \ldots, q_{n}$, the variational functional can be written as

$$
A=\frac{1}{2} \sum_{i, j} \eta_{i j} \dot{q}_{i} \dot{q}_{j}+\sum_{i=1}^{n} \frac{\partial F\left(q_{1}, \ldots, q_{n}\right)}{\partial q_{i}} \dot{q}_{i}
$$

where in the case of the $q_{i}$ being field variables, the summation should be replaced by integrals, and partial derivatives by functional derivatives. Here $\eta_{i j}$ are the damping coefficients, $\dot{q}_{i}$ are the rates corresponding to $q_{i}, \frac{1}{2} \sum_{i, j} \eta_{i j} \dot{q}_{i} \dot{q}_{j}$ is the so-called dissipation function which is half of the rate of energy dissipation, and $F\left(q_{1}, \ldots, q_{n}\right)$ is the free energy function. Note that the damping coefficients $\eta_{i j}$ must be symmetric with respect to the interchange of the two indices, as required by microscopic reversibility. Minimizing $A$ with respect to the rates $\left\{\dot{q}_{i}\right\}$, for prescribed $\left\{q_{i}\right\}$, yields for $i=1, \ldots, n$ the equations

$$
\sum_{j=1}^{n} \eta_{i j} \dot{q}_{j}=-\frac{\partial F\left(q_{1}, \ldots, q_{n}\right)}{\partial q_{i}}
$$

which describe the balance of the conservative forces due to the free energy gradients and the dissipative forces that are linear in rates.

\subsection{Surface layer and the natural boundary conditions}

A surface layer of fluid exists under the influence of the solid wall potential, which is physically the source of the boundary conditions which describe the effects of the solid surface on the bulk fluid. Consider the fluid to be in the half-space $z \geqslant 0$. Since the molecular interactions are always characterized by a finite range in which there can be a repulsive component that delineates the 'size' of the molecules, it is inevitable that there be a finite range within which the wall effect on the fluid is fully realized. It follows that there is a plane from which the fluid-solid interaction energy accumulates starting from zero, so that the tangential stress is zero and fluid-solid interfacial tension effect is also absent at this plane, denoted $z=0$. Both effects take a small but finite distance to be fully realized (shown below through molecular dynamics simulations), and this defines what we mean by the 'surface layer'. Natural boundary conditions are simply the dynamic and force balance consequences at $z=0$ that follow from zero tangential stress and zero fluid-solid interfacial tension. Physically the $z=0$ plane may coincide with the centres of mass of the solid molecules, as is made clear in the following considerations.

The interaction energy density between the fluid molecules and the solid wall may be characterized by $V_{w}(z) n(z)$, where $V_{w}(z)$ denotes the wall potential that has a finite range $h$, and $n(z)$ is the fluid molecular density. In the immediate vicinity of the wall molecules, the repulsive nature of the wall potential (where $V_{w}(z)$ is positive) grows very fast as $z$ approaches zero (the centres of mass of the solid molecules), but the product $V_{w}(z) n(z) \rightarrow 0$ since $n(z) \sim \exp \left[-V_{w}(z) / k_{\mathrm{B}} T\right]$ decreases even faster. Moreover, since $V_{w}(z)$ has a finite range, $V_{w}(z) n(z) \rightarrow 0$ as $z$ increases beyond $h$. From these considerations it is clear that $V_{w}(z) n(z)$ is a peaked function of $z$ with a finite width $\sim h$. This is the surface layer, as illustrated in figure 1 .

The wall potential is characterized by $(a)$ a plane where the fluid-solid interaction energy density is zero, and $(b)$ a geometric structure factor in the $(x, y)$-plane, plus a saturation distance perpendicular to the $(x, y)$-plane that is on the order of the molecular interaction distance, i.e. on the order of a few Angstroms at most. For (a), the immediate consequence is the so-called natural boundary condition of zero tangential stress at $z=0$. This point will be made precise in the following section. 


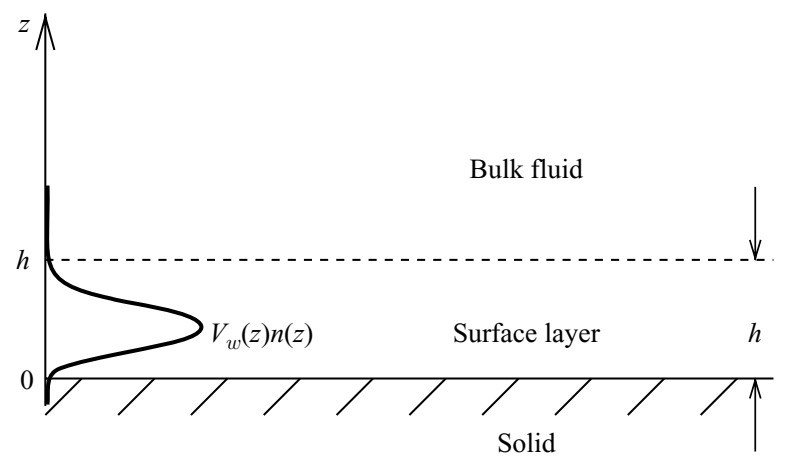

FIgURE 1. A schematic illustration of the surface layer that extends from $z=0$ to $h$. The solid curve denotes the interaction energy density $V_{w}(z) n(z)$ between the fluid molecules and the solid wall as a peaked function of $z$.

For $(b)$, it should be emphasized that for the concept of a hydrodynamic boundary condition to be valid, the surface-layer thickness must be the smallest length scale in the problem. Physically, the surface-layer thickness is comparable to the range of short-range repulsive forces that usually operate on an atomic scale. In particular, surface layer as defined above is much thinner than the precursor film thickness, whose existence is due to the longer-range component of the wall potential, i.e. the van der Waal interaction (de Gennes 1985). Thus it is valid to use hydrodynamic boundary condition(s) in the treatment of precursor films, with long-range van der Waals forces explicitly taken into account in the momentum equation. Work is currently under way in this direction. However, it cannot be excluded that there exist some instances (for example, due to the nature of the wall molecules, e.g. polymers), where the surface layer thickness may become comparable to other scale(s) in the problem. For these cases, the conventional concept of boundary conditions may have to be revised. In the present scaling approach, the hydrodynamic boundary conditions are obtained by keeping the leading-order terms of the equations of motion in the surface layer, that are independent of the surface layer thickness $h$. An expansion in $h$ to the next-order terms would yield information regarding the accuracy of the hydrodynamic boundary conditions in replacing the surface layer dynamics.

To identify the surface layer, we note that fluid-solid interaction can give rise to two distinct physical quantities: the tangential frictional force exerted by the wall on the fluid and the fluid-solid interfacial tension. In terms of the Onsager principle, the former is related to the dissipation function while the latter is part of the free energy. In particular, in the GNBCs that resolve the moving contact line problem (Qian et al. 2006), these are the two quantities solely contributed by the surface layer.

In the regime of linear friction, the tangential wall force per unit area exerted on the fluid is proportional to the slip velocity, i.e. $G_{x}^{w}=-\beta v_{x}$, where $G_{x}^{w}$ is the tangential wall force per unit area and $v_{x}$ the tangential slip velocity, with the solid surface assumed to be still and in the $(x, y)$-plane and the fluid in the half-space $z \geqslant 0$. The slip coefficient $\beta$, which is the friction coefficient of the fluid-solid interface, arises from the lateral inhomogeneity (corrugation) of the structured solid wall. (We consider a fluid bounded by an atomically smooth solid surface. By atomically smooth, we mean for example a dense plane of a perfect crystalline lattice.) Away from the fluid-solid boundary $(z=0)$, each fluid molecule can interact with many 

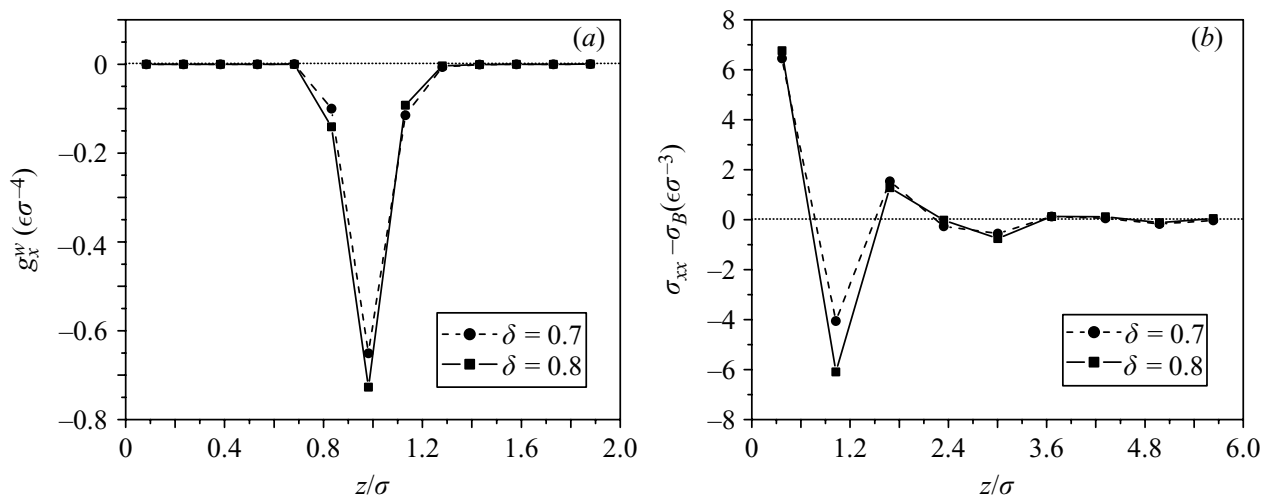

FIGURE 2. Molecular dynamics evidence for the existence of a surface layer. For technical details of our simulations, see Appendix A. (a) The tangential wall force density $g_{x}^{w}$ plotted as a function of distance $z$ away from the solid surface, with $z=0$ corresponding to the centre of the first layer of solid atoms in the bottom wall. Here the tangential wall force density was measured in a standard non-equilibrium MD simulation of the Couette flow confined between two planar solid surfaces, by subdividing the surface layer into many thin sections. It is seen that the force density $g_{x}^{w}$ is distributed in a very narrow range. The tangential wall force per unit area, $G_{x}^{w}=\int \mathrm{d} z g_{x}^{w}(z)$, is obtained by integrating $g_{x}^{w}$ across the surface layer. The slip velocity $v_{x}$ is defined as the average tangential velocity of the fluid in the range of tangential wall force, measured relative to the bottom wall. We found $G_{x}^{w}=-0.136 \epsilon \sigma^{-3}$ and $v_{x}=0.102(\epsilon / m)^{1 / 2}$ for $\delta=0.7$, and $G_{x}^{w}=-0.144 \epsilon \sigma^{-3}$ and $v_{x}=0.0755(\epsilon / m)^{1 / 2}$ for $\delta=0.8$, from which we obtained $\beta=1.33(\epsilon m)^{1 / 2} \sigma^{-3}$ for $\delta=0.7$ and $\beta=1.91(\epsilon m)^{1 / 2} \sigma^{-3}$ for $\delta=0.8$ using $G_{x}^{w}=-\beta v_{x}$. The observed increase of $\beta$ with increasing fluid-solid attractive interaction agrees with the theoretical prediction of Barrat \& Bocquet (1999). (b) Time-averaged profile of the stress anisotropy measured in an equilibrium MD simulation. Here $\sigma_{x x}=\left(\sigma_{x x}+\sigma_{y y}\right) / 2$ is the parallel component of the stress associated with the fluid-solid interface, and $\sigma_{B}$ represents the uniform normal component of the stress $\sigma_{z z}$ which is equal to $\sigma_{x x}$ in the bulk where the stress is isotropic. The interfacial tension $\gamma_{\mathrm{FS}}$ can be obtained by integrating $\sigma_{x x}-\sigma_{B}$ across the fluid-solid interface, $\gamma_{\mathrm{FS}}=\int \mathrm{d} z\left[\sigma_{x x}(z)-\sigma_{B}\right]$ (for more details, see Appendix B). We found $\gamma_{\mathrm{FS}}=2.04 \epsilon \sigma^{-2}$ for $\delta=0.7$ and $\gamma_{\mathrm{FS}}=0.91 \epsilon \sigma^{-2}$ for $\delta=0.8$. It is seen that $\gamma_{\mathrm{FS}}$ decreases with increasing fluid-solid attraction.

solid atoms on a nearly equal basis. Thus, as experienced by the fluid molecules, the periodic corrugation amplitude of the wall potential would quickly decrease with increasing distance from the solid surface. Hence the tangential wall force tends to saturate at a relatively short range. This can be seen from molecular dynamics (MD) simulation results shown in figure 2(a), which clearly indicate the tangential wall force density $g_{x}^{w}$ (with $G_{x}^{w}=\int \mathrm{d} z g_{x}^{w}(z)$ ) to be in the form of a sharply peaked function approximately one molecular distance away from the centre of the first layer of solid atoms. In contrast, the normal component of the wall force arises from the fluid-solid interaction directly, independent of whether the wall potential is periodic or not. Consequently, the normal wall force saturates slower than the tangential component. Physically, the fluid-solid interfacial tension $\gamma_{\mathrm{FS}}$ is contributed by the normal wall force (with the atomic-scale lateral inhomogeneities of the solid surface smoothed out, see Appendix B). We have carried out MD simulations showing the finite range over which $\gamma_{\mathrm{FS}}$ is defined, in accordance with the Kirkwood-Buff formula for interfacial tension (Kirkwood \& Buff 1949). The results are shown in figure $2(b)$. Again, the interfacial tension saturates within a finite but small distance away from the centre of the first layer of solid atoms. But this distance is larger than that seen in 
figure 2(a) for the tangential component of fluid-solid interaction. The surface layer thickness $h$ is taken to be the larger of the two. In the phenomenological continuum hydrodynamics presented in $\S 2.3$, the tangential wall force per unit area $G_{w}^{x}$ and the fluid-solid interfacial free energy per unit area $\gamma_{\mathrm{FS}}$ will be realized by introducing their corresponding density quantities into the surface layer.

In a recent review article by Bocquet \& Barrat (2007) on flow boundary conditions, it has been pointed out that a slip boundary condition is physically a constitutive relation involving the fluid-solid interface, comparable to that of the bulk equations in every respect. This is completely in line with the physics considerations behind the introduction of a surface layer. More specifically, the concept of a surface layer is clearly manifest in the Kubo-like formula derived for the slip coefficient (Barrat \& Bocquet 1999), similar to the Green-Kubo relation for the shear viscosity. The corresponding autocorrelation function is contributed by the first layer of fluid. It can be shown that $\beta$ is proportional to the integrated quantity $\int_{0}^{\infty} \mathrm{d} z \rho(z) V_{\mathrm{FS}}(z)^{2}$, where $\rho(z)$ is the density profile of the fluid perpendicular to the solid surface and $V_{\mathrm{FS}}(z)$ is the amplitude of potential corrugation. Since $\rho(z)$ vanishes quickly toward zero due to the short-range repulsive interaction, while $V_{\mathrm{FS}}(z)$ decreases quickly with increasing distance from the solid surface, the product $\rho(z) V_{\mathrm{FS}}(z)^{2}$ must be sharply peaked in the surface layer, in agreement with the non-equilibrium MD observation for the tangential wall force depicted in figure 2(a) (Qian et al. 2003).

In the following, we wish to treat the surface layer in the continuum sense. This is possible provided the variables in the continuum formulation are the time- and fluidmolecules-averaged values. This is the usual manner in which the underlying discrete nature of fluid and solid is made to correspond with the continuum formulations. Thus we may regard the fluid molecules in the surface layer as constituting a two-dimensional fluid, where the lateral inhomogeneities are homogeneized over the hydrodynamic length scale. It is over this continuum hydrodynamic length scale that the plane of zero fluid-solid interaction energy density is introduced, with atomicscale inhomogeneities smoothed out (the effect of these inhomogeneities is taken into account in the evaluation of the slip coefficient $\beta$, as described above). This approach follows the conventional continuum description deduced from molecular dynamics.

In essence, our approach is to introduce the continuum description within a surface layer of finite thickness. And the hydrodynamic equations of motion within this surface layer are derived from the Onsager principle, taking into account the fluidsolid interactions. A scaling approach is then introduced to preserve the integrated effect of the surface layer on the bulk hydrodynamics as its thickness $h$ is continuously reduced towards $0^{+}$. Hydrodynamic boundary conditions emerge as a consequence.

However, it should be noted that by using the continuum description and the Onsager principle, we obtain only the form(s) of hydrodynamic boundary conditions. The relevant fluid-solid interfacial physics is contained in the phenomenological parameters that appear in the hydrodynamic boundary conditions. The evaluation of these parameters requires the application of statistical mechanics and kinetic theory to a detailed interfacial model that includes both geometric information as well as molecular interactions. These are beyond the scope of the present work, the purpose of which is only to provide a general framework for deriving the form of hydrodynamic boundary conditions. In this context it is accurate to regard the hydrodynamic boundary conditions as just another manifestation of linear response phenomena in dissipative systems, e.g. electrical current is linearly proportional to voltage difference, flow rate through a porous medium is linearly proportional to pressure difference, heat flow is linearly proportional to temperature gradient, etc., all of which may be traced 


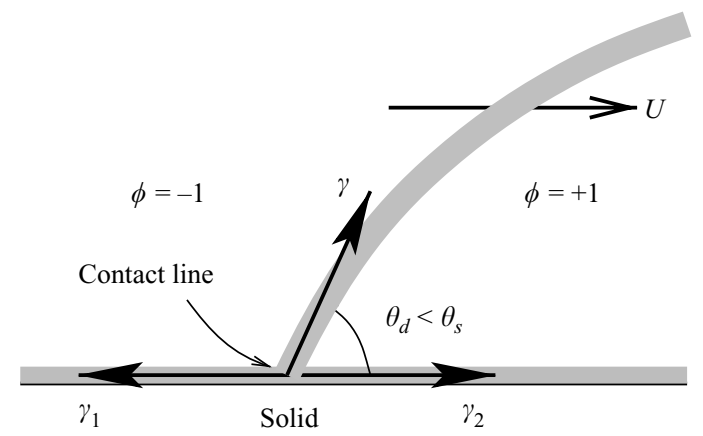

FIGURE 3. A schematic illustration for the moving contact line in immiscible two-phase flows. The two fluids are represented by the phase fields $\phi=-1$ and $\phi=+1$, respectively. The fluid-fluid interface intersects the solid wall at the contact line which joins three interfaces - one fluid-fluid interface and two fluid-solid interfaces. Each interface is characterized by an interfacial tension, with $\gamma$ for the fluid-fluid interface and $\gamma_{1(2)}$ for the fluid-solid interfaces. Owing to the contact-line motion relative to the solid wall, the dynamic contact angle $\theta_{d}$ deviates from the static contact angle $\theta_{s}$, which is determined by the Young equation $\gamma \cos \theta_{s}+\gamma_{2}=\gamma_{1}$. Each interface is shaded with a finite width to indicate the diffuse nature of the fluid-fluid interface and the surface layer.

back to the Onsager principle. In all these cases the evaluation of the coefficients of proportionality, i.e. electrical conductivity, permeability, heat conductivity, etc., has to be carried out separately for each material system, and may depend on microstructure and other detailed information. The purpose of the present work is to provide the missing link - the scaling approach - which connects the Onsager principle to the hydrodynamic boundary conditions by recognizing the physical existence of a surface layer. The evaluation of the relevant phenomenological coefficients is left as a future task.

\subsection{Variational derivation of the equations of motion and natural boundary conditions}

The governing equations for fluid motion, from the surface layer to the bulk, can be derived from the Onsager variational principle with the consideration that wall potential is of a small but finite extent, i.e. the relevant wall potential can be treated as negligible outside the surface layer. In this section, we treat the case of two-phase immiscible flows that involve a moving contact line, defined as the intersection of the fluid-fluid interface with the solid wall (see figure 3). To distinguish between the two immiscible fluids, a phase field $\phi(\boldsymbol{r})$ is introduced to measure the local relative concentration (Cahn \& Hilliard 1958; Seppecher 1996; Jacqmin 2000; Chen, Jasnow \& Vinals 2000; Pismen \& Pomeau 2000; Briant \& Yeomans 2004). $\phi(\boldsymbol{r})$ varies continuously from the value of -1 on the fluid 1 side to the value of +1 on the fluid 2 side, with a sharp variation across the fluid-fluid interfacial region. Since $\phi$ is a locally conserved quantity, it satisfies the continuity equation $\dot{\phi}=\partial \phi / \partial t+\boldsymbol{v} \cdot \nabla \phi=-\nabla \cdot \boldsymbol{J}$, where $\boldsymbol{J}$ denotes the diffusive current density, and $\boldsymbol{v}$ the local fluid velocity. The velocity $\boldsymbol{v}$, which appears in the momentum equation, is defined to be the barycentric, or mass-averaged, velocity $\boldsymbol{v}=\sum_{i} \rho_{i} \boldsymbol{v}_{i} / \rho$, where $\rho_{i}$ is the position-dependent mass density of species $i, \boldsymbol{v}_{i}$ is the position-dependent velocity of species $i$, and $\rho=\sum_{i} \rho_{i}$ is the total mass density. The diffusive current density (flux) of particles of type $i$, $\boldsymbol{J}_{i}=\rho_{i}\left(\boldsymbol{v}_{i}-\boldsymbol{v}\right.$ ), is defined relative to the barycentric velocity (de Groot \& Mazur 1984). Note that for binary fluids, $\boldsymbol{J}_{1}+\boldsymbol{J}_{2}=\sum_{i} \rho_{i} \boldsymbol{v}_{i}-\rho \boldsymbol{v}=0$, and therefore only one diffusive current density is used. 


\subsubsection{Dissipation function}

We start from the dissipation function $\Phi(\dot{\alpha}, \dot{\alpha})$ that is quadratic in the rates:

$$
\Phi=\Phi_{v}+\Phi_{s}+\Phi_{d},
$$

in which $\Phi_{v}, \Phi_{s}$, and $\Phi_{d}$ are due to shear viscosity, wall friction, and diffusion, respectively, given by

$$
\begin{gathered}
\Phi_{v}=\frac{1}{2} \int \mathrm{d} \boldsymbol{r}\left[\frac{\eta}{2}\left(\partial_{i} v_{j}+\partial_{j} v_{i}\right)^{2}\right], \\
\Phi_{s}=\frac{1}{2} \int \mathrm{d} \boldsymbol{r}\left[\bar{\beta} \boldsymbol{v}_{\tau}^{2}\right], \\
\Phi_{d}=\frac{1}{2} \int \mathrm{d} \boldsymbol{r}\left[\frac{\boldsymbol{J}^{2}}{M}\right],
\end{gathered}
$$

where $\eta$ is the shear viscosity, $v_{i}$ the components of $\boldsymbol{v}$, and $\bar{\beta}$ is the slip coefficient (whose value vanishes beyond the surface layer) that owes its origin to the tangential component of the dynamic stress exerted by the wall on fluid molecules (Barrat \& Bocquet 1999; Qian et al. 2003), $\boldsymbol{v}_{\tau}$ is the projection of $\boldsymbol{v}$ on the solid surface, and $M$ is the mobility coefficient. Owing to the influence of the wall, values of $\eta$ and $M$ near the solid surface may deviate from their bulk values. Below we assume that the planar solid surface is in the $(x, y)$-plane, the fluids are in the half-space $z \geqslant 0$, and for generality $\eta, \bar{\beta}$, and $M$ are all regarded as functions of $z$, with $\eta$ and $M$ approaching their bulk values and $\bar{\beta} \rightarrow 0$ for $z>h$. The parameters $\eta, \bar{\beta}$, and $M$ all locally depend on $\phi$, i.e. $\eta=\eta(z ; \phi), \bar{\beta}=\bar{\beta}(z ; \phi)$, and $M=M(z ; \phi)$. Physically, $\Phi_{s}$ represents the dissipation due to the viscous coupling between the fluid and wall. The corresponding dissipative force is $-\bar{\beta} \boldsymbol{v}_{\tau} \equiv-\bar{\beta} \boldsymbol{v} \cdot(\mathbf{1}-\hat{z} \hat{z})$, the tangential wall force density (whose $x$ component is $g_{x}^{w}$ introduced in $\S 2.2$ ).

\subsubsection{Free energy components}

The free energy function $F[\phi(\boldsymbol{r})]$ consists of the Cahn-Hilliard $(\mathrm{CH})$ free energy $F_{\mathrm{CH}}[\phi(\boldsymbol{r})]$ which stabilizes the fluid-fluid interface between the two immiscible fluids (Cahn \& Hilliard 1958) and the surface free energy $F_{\mathrm{FS}}[\phi(\boldsymbol{r})]$ which arises from the fluid-solid interaction (Thompson \& Robbins 1989):

$$
F[\phi(\boldsymbol{r})]=F_{\mathrm{CH}}[\phi(\boldsymbol{r})]+F_{\mathrm{FS}}[\phi(\boldsymbol{r})],
$$

in which $F_{\mathrm{CH}}[\phi(\boldsymbol{r})]$ and $F_{\mathrm{FS}}[\phi(\boldsymbol{r})]$ are given by

$$
\begin{gathered}
F_{\mathrm{CH}}[\phi(\boldsymbol{r})]=\frac{3 \gamma}{2 \sqrt{2}} \int \mathrm{d} \boldsymbol{r}\left[\frac{\xi}{2}(\nabla \phi)^{2}+\frac{1}{4 \xi}\left(\phi^{2}-1\right)^{2}\right], \\
F_{\mathrm{FS}}[\phi(\boldsymbol{r})]=\int \mathrm{d} \boldsymbol{r}\left[\bar{\gamma}_{\mathrm{FS}}(z ; \phi)\right] .
\end{gathered}
$$

Here $\gamma$ and $\xi$ are the interfacial tension and thickness of the fluid-fluid interface, and $\bar{\gamma}_{\mathrm{FS}}(z ; \phi)$ represents the energy density for the fluid-solid interaction that extends to a distance $h$ and locally depends on $\phi$. Physically, this energy density arises from the wall potential $V_{w}$ introduced in $\S 2.2$. The relation between $\bar{\gamma}_{\mathrm{FS}}$ and $V_{w}$ is presented in Appendix B. The rate of change of $F[\phi(r)]$ can be expressed by

$$
\dot{F}[\phi(\boldsymbol{r})]=\int \mathrm{d} \boldsymbol{r}\left[\mu_{\mathrm{CH}} \frac{\partial \phi}{\partial t}\right]+\int \mathrm{d} S\left[L_{\mathrm{CH}} \frac{\partial \phi}{\partial t}\right]+\int \mathrm{d} \boldsymbol{r}\left[\frac{\partial \bar{\gamma}_{\mathrm{FS}}}{\partial \phi} \frac{\partial \phi}{\partial t}\right],
$$


in which

$$
\mu_{\mathrm{CH}}=\frac{3 \gamma}{2 \sqrt{2}}\left[-\xi \nabla^{2} \phi+\frac{1}{\xi} \phi\left(\phi^{2}-1\right)\right]
$$

is the chemical potential and

$$
L_{\mathrm{CH}}=-\frac{3 \gamma \xi}{2 \sqrt{2}} \partial_{z} \phi,
$$

with $\int \mathrm{d} S$ denoting the surface integral in the plane of $z=0$. Substituting the material derivative $\dot{\phi}=\partial \phi / \partial t+\boldsymbol{v} \cdot \nabla \phi$ and the continuity equation $\dot{\phi}=-\nabla \cdot \boldsymbol{J}$ into the expression for $\dot{F}[\phi(\boldsymbol{r})]$ yields

$$
\begin{aligned}
\dot{F}[\phi(r)]= & \int \mathrm{d} \boldsymbol{r}\left[\nabla \mu_{\mathrm{CH}} \cdot \boldsymbol{J}-\mu_{\mathrm{CH}}(\boldsymbol{v} \cdot \nabla \phi)\right] \\
& +\int \mathrm{d} S\left[L_{\mathrm{CH}}(\dot{\phi}-\boldsymbol{v} \cdot \nabla \phi)\right] \\
& +\int \mathrm{d} \boldsymbol{r}\left[\nabla\left(\frac{\partial \bar{\gamma}_{\mathrm{FS}}}{\partial \phi}\right) \cdot \boldsymbol{J}-\frac{\partial \bar{\gamma}_{\mathrm{FS}}}{\partial \phi}(\boldsymbol{v} \cdot \nabla \phi)\right],
\end{aligned}
$$

in which the impermeability boundary condition $J_{z}=0$ has been used at the solid surface such that $\int \mathrm{d} r[f \dot{\phi}]=\int \mathrm{d} r[\nabla f \cdot \boldsymbol{J}]$ with $f=\mu_{\mathrm{CH}}$ or $\partial \bar{\gamma}_{\mathrm{FS}} / \partial \phi$.

\subsubsection{Variational functional and Euler-Lagrange equations}

According to the Onsager principle, the sum of $\Phi$ and $\dot{F}$ gives the action functional $A$ :

$$
\begin{aligned}
A[\boldsymbol{v}(\boldsymbol{r}), \boldsymbol{J}(\boldsymbol{r}), \dot{\phi}(\boldsymbol{r})]= & \int \mathrm{d} \boldsymbol{r}\left[\frac{\eta}{4}\left(\partial_{i} v_{j}+\partial_{j} v_{i}\right)^{2}\right]+\int \mathrm{d} \boldsymbol{r}\left[\frac{\bar{\beta}}{2} \boldsymbol{v}_{\tau}^{2}\right]+\int \mathrm{d} \boldsymbol{r}\left[\frac{\boldsymbol{J}^{2}}{2 M}\right] \\
& +\int \mathrm{d} \boldsymbol{r}\left[\nabla \mu_{\mathrm{CH}} \cdot \boldsymbol{J}-\mu_{\mathrm{CH}}(\boldsymbol{v} \cdot \nabla \phi)\right]+\int \mathrm{d} S\left[L_{\mathrm{CH}}(\dot{\phi}-\boldsymbol{v} \cdot \nabla \phi)\right] \\
& +\int \mathrm{d} \boldsymbol{r}\left[\nabla\left(\frac{\partial \bar{\gamma}_{\mathrm{FS}}}{\partial \phi}\right) \cdot \boldsymbol{J}-\frac{\partial \bar{\gamma}_{\mathrm{FS}}}{\partial \phi}(\boldsymbol{v} \cdot \nabla \phi)\right],
\end{aligned}
$$

which is to be minimized with respect to the rates $\{\boldsymbol{v}, \boldsymbol{J}, \dot{\phi}\}$, supplemented with the incompressibility condition $\nabla \cdot \boldsymbol{v}=0$. Considering that $\phi$ is conserved in the bulk but not at the solid surface, we choose $J$ as the rate variable in the bulk and $\dot{\phi}$ the rate variable at the surface $z=0$. Minimization with respect to $v$ in the bulk yields the variational form

$$
\begin{aligned}
\int \mathrm{d} \boldsymbol{r}\left[-\eta \partial_{j}\left(\partial_{j} v_{i}+\partial_{i} v_{j}\right) \delta v_{i}\right]+\int \mathrm{d} \boldsymbol{r}\left[\bar{\beta} \boldsymbol{v}_{\tau} \cdot \delta \boldsymbol{v}_{\tau}\right] \\
+\int \mathrm{d} \boldsymbol{r}\left[-\left(\mu_{\mathrm{CH}}+\frac{\partial \bar{\gamma}_{\mathrm{FS}}}{\partial \phi}\right) \partial_{i} \phi \delta v_{i}\right]+\int \mathrm{d} \boldsymbol{r}\left[-\partial_{i} \lambda \delta v_{i}\right]=0
\end{aligned}
$$

with $\lambda$ a Lagrange multiplier for $\nabla \cdot v=0$. From equation (2.15) we obtain the Stokes equation

$$
-\nabla p+\nabla \cdot \boldsymbol{\sigma}^{v}+\left(\mu_{\mathrm{CH}}+\frac{\partial \bar{\gamma}_{\mathrm{FS}}}{\partial \phi}\right) \nabla \phi-\bar{\beta} \boldsymbol{v} \cdot(\mathbf{1}-\hat{z} \hat{z})=0,
$$

where $p=-\lambda$ is identified as the pressure and $\sigma^{v}=\eta\left[\nabla v+(\nabla v)^{T}\right]$ the Newtonian viscous stress tensor. The last term on the left-hand side of equation (2.16) is the tangential wall force density arising from the viscous coupling between the fluid and 
wall, localized to the surface layer as illustrated in figure $2(a)$. This treatment of the friction between the fluid and wall as being spatially distributed in a thin layer is in fact the distinguishing feature of the present phenomenological approach. It is interesting to note that equation (2.16) is similar to the Darcy-Brinkman equation (Brinkman 1947) for flow in a dilute porous medium, with an anisotropic permeability that is spatially inhomogeneous. In particular, the slip coefficient $\bar{\beta}$ plays the role of an inverse permeability that is localized to the surface layer and controls the tangential momentum transport between the fluid and wall. Similar minimization with respect to $\boldsymbol{J}$ yields the constitutive relation

$$
\boldsymbol{J}=-M \nabla\left(\mu_{\mathrm{CH}}+\frac{\partial \gamma_{\mathrm{FS}}}{\partial \phi}\right),
$$

which combines with the continuity equation for the $\phi$ to yield the convectiondiffusion equation

$$
\dot{\phi}=\frac{\partial \phi}{\partial t}+\boldsymbol{v} \cdot \nabla \phi=\nabla \cdot\left[M \nabla\left(\mu_{\mathrm{CH}}+\frac{\partial \gamma_{\mathrm{FS}}}{\partial \phi}\right)\right] .
$$

To derive the relevant boundary conditions, it is observed that minimization of the action functional, equation (2.14), with respect to $v$ at the surface $z=0$ yields the variational form

$$
\int \mathrm{d} S\left[m_{j} \eta\left(\partial_{j} v_{i}+\partial_{i} v_{j}\right) \delta v_{i}\right]+\int \mathrm{d} S\left[-L_{\mathrm{CH}} \partial_{i} \phi \delta v_{i}\right]=0
$$

with $v_{i}$ restricted to the $(x, y)$-plane and $\boldsymbol{m}=-\hat{z}$ the outward pointing unit vector normal to the solid surface. From equation (2.19) we obtain the boundary condition

$$
\hat{z} \cdot \sigma \cdot(\mathbf{1}-\hat{z} \hat{z})=0,
$$

where $\sigma=\sigma^{v}+\sigma^{Y}, \sigma^{v}$ being the viscous stress and $\sigma^{Y}=-3 \gamma \xi /(2 \sqrt{2}) \nabla \phi \otimes \nabla \phi$ the Young stress. In addition, minimization with respect to $\dot{\phi}$ at the surface yields

$$
L_{\mathrm{CH}}=0
$$

(and thus $\partial_{z} \phi=0$, see equation (2.12)), necessary for the 'chemical' equilibrium at the solid surface. Therefore

$$
\hat{z} \cdot \boldsymbol{\sigma}^{v} \cdot(\mathbf{1}-\hat{z} \hat{z})=0
$$

and

$$
\hat{z} \cdot \boldsymbol{\sigma}^{Y} \cdot(\mathbf{1}-\hat{z} \hat{z})=0,
$$

separately. Equations (2.21) and (2.22) simply reflect the fact that there is no fluid beyond $z=0$ and are denoted 'natural' boundary conditions, as illustrated in figure 4 . In particular, the natural boundary conditions imply the contact angle at $z=0$ to be $90^{\circ}$, since the absence of wall potential means that there is no fluid-solid interfacial tension at the $z=0$ plane. It is worth emphasizing that the natural boundary condition of zero tangential stress at $z=0$ results from the fact that the tangential momentum transport between the fluid and wall occurs in the surface layer, where the tangential wall force $-\bar{\beta} \boldsymbol{v} \cdot(\mathbf{1}-\hat{z} \hat{z})$ is narrowly distributed. The natural boundary conditions are supplemented by the impenetrability conditions $v_{z}=0$ and $J_{z}=0$.

Given the equations of motion (2.16) and (2.18), supplemented by the natural boundary conditions and the impenetrability conditions, the hydrodynamic solution in the $z>0$ domain is well-defined. However, such a solution necessarily requires the 


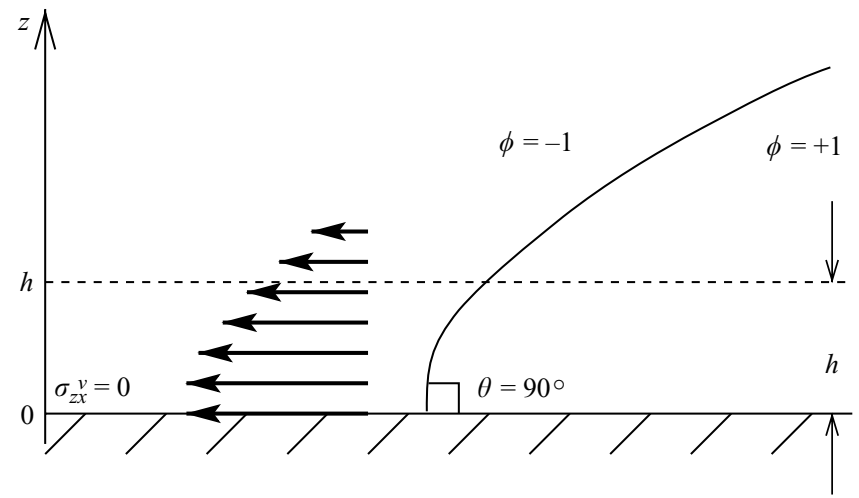

FiguRE 4. A schematic illustration for the natural boundary conditions $\hat{z} \cdot \boldsymbol{\sigma}^{v} \cdot(\mathbf{1}-\hat{z} \hat{z})=0$ and $\hat{z} \cdot \sigma^{Y} \cdot(1-\hat{z} \hat{z})=0$. The former is illustrated by a flow field with $\sigma_{z x}^{v}=\eta \partial_{z} v_{x}=0$ and the latter by a fluid-fluid interface with a $90^{\circ}$ contact angle at $z=0$. Note that this contact angle is different from the contact angle $\theta_{d(s)}$ illustrated in figure 3, which is defined at $z=h$.

resolution of the surface layer, which would be numerically impractical in most cases. The purpose of the following section is to demonstrate that to leading order of the surface-layer thickness $h$, treated as a small quantity in units where the $z$-dimension of the system is taken to be 1 , the effects of the surface layer can be captured by the hydrodynamic boundary conditions.

\section{Scaling and the hydrodynamic boundary conditions}

Boundary conditions may be regarded as the hydrodynamic consequences of the surface layer on the bulk fluid, without the necessity of resolving the surface layer. Derivation of the boundary conditions may be accomplished in two ways, both presented below. The first is to integrate the surface layer action functional (under the influence of the wall potential) so as to represent its effects on the bulk at the surface layer - bulk interface. The second is to have a mathematical limiting process in which the effects of the surface layer hydrodynamics are kept constant while letting $h$ approach $0^{+}$. We show in the following that in order for both approaches to be viable in a consistent manner, the parameters and variables that affect or appear in the boundary conditions should be independent of $h$, a constraint which must be consistently satisfied. We denote the relations of the various surface layer parameters/variables with respect to $h$ the 'scaling relations'. They have the physical meaning of relating the surface layer parameters/variables to those appearing in the boundary conditions. When the scaling relations are satisfied, mathematically the surface layer hydrodynamics exhibits $h$-independence to the leading order of $h$, thus making possible the definition of boundary conditions.

For simplicity, below we consider the case of two-dimensional flows with $\phi=\phi(x, z, t)$ and $\boldsymbol{v}=v_{x}(x, z, t) \hat{\boldsymbol{x}}+v_{z}(x, z, t) \hat{z}$ such that $\boldsymbol{v} \cdot(\mathbf{1}-\hat{z} \hat{z})=v_{x} \hat{\boldsymbol{x}}, \hat{z} \cdot \boldsymbol{\sigma}^{v} \cdot(\mathbf{1}-$ $\hat{z} \hat{z})=\sigma_{z x}^{v} \hat{\boldsymbol{x}}$, and $\hat{z} \cdot \sigma^{Y} \cdot(\mathbf{1}-\hat{z} \hat{z})=\sigma_{z x}^{Y} \hat{\boldsymbol{x}}$. Generalization to the three-dimensional case is straightforward.

\subsection{Scaling relations}

We obtain the scaling relations by first requiring the z-integrated material properties that owe their origin to the wall potential be independent of $h$, and then demanding 
consistency with other parameters/variables through the equations of motion and related expressions.

\subsubsection{Scaling and surface-layer thickness variation}

In what follows we deduce the scaling relations, i.e. variation of the various surface layer parameters and variables with respect to $h$. Here the $h$ variation should be interpreted as follows. Consider a series of replicas of the physical system in which the surface layer thickness $h$ is monotonically decreased, and the first copy of the replica series represents the physical system. We require the bulk hydrodynamics to stay the same, to the leading order of $h$, for every replica in the series. Provided this requirement can be met, then the boundary conditions are precisely those which appear at $z=h$, at the end of the replica series as $h \rightarrow 0^{+}$. Since the dynamics of the surface layer is transmitted to the bulk via its interface with the bulk, the requirement that the bulk hydrodynamics stays the same in every replica may be satisfied by making what appears at $z=h$ to be $h$-independent. In the following we show that by first requiring the constancy of $\beta$ and $\gamma_{\mathrm{FS}}$, which represent integrated wall effects on the fluid, and then demanding consistency of $h$ variation in the surface layer equations of motion, a series of scaling relations may be obtained. Using such scaling relations, the Onsager functional can be written in a form that takes into account only the integrated effects of the surface layer and therefore is independent of $h$. Variation of the Onsager functional then yields the conditions at $z=h$. Since these conditions are $h$-independent, they are precisely the boundary conditions. In this way we deduce what should be defined mathematically as the boundary conditions to correspond exactly with what appears at $z=h$ for the first copy of the replica series, which is the physical system.

\subsubsection{Scaling of $\bar{\beta}, \bar{\gamma}_{\mathrm{FS}}$ and the chemical potential}

We start by proposing that the material properties $\bar{\beta}=\bar{\beta}(z ; \phi)$ and $\bar{\gamma}_{\mathrm{FS}}=\bar{\gamma}_{\mathrm{FS}}(z ; \phi)$ are distributed along the $z$-direction according to

$$
\bar{\beta}=\frac{1}{h} D_{\beta}(z / h ; \phi) \beta(\phi)
$$

and

$$
\bar{\gamma}_{\mathrm{FS}}=\frac{1}{h} D_{\gamma}(z / h ; \phi) \gamma_{\mathrm{FS}}(\phi),
$$

where $D_{\beta}$ and $D_{\gamma}$ satisfy the normalization conditions

$$
\int_{0}^{h} \mathrm{~d} z\left[\frac{1}{h} D_{\beta}(z / h ; \phi)\right]=1
$$

and

$$
\int_{0}^{h} \mathrm{~d} z\left[\frac{1}{h} D_{\gamma}(z / h ; \phi)\right]=1
$$

(with $\phi$ regarded as a constant in integration). These two scaling relations are required by the physical condition that the surface-layer-integrated properties $\beta(\phi)=\int_{0}^{h} \mathrm{~d} z[\bar{\beta}(z ; \phi)]$ and $\gamma_{\mathrm{FS}}(\phi)=\int_{0}^{h} \mathrm{~d} z\left[\bar{\gamma}_{\mathrm{FS}}(z ; \phi)\right]$ should be independent of $h$. We note that $\bar{\beta} v_{x}$ is the frictional body force density due to the wall and $\beta v_{x}$ is the 
frictional force per unit area of the solid surface if $v_{x}$ is nearly constant across the surface layer (shown below). Similarly, we note that $\gamma_{\mathrm{FS}}(\phi)$ is the fluid-solid interfacial free energy per unit area if $\phi$ is nearly constant across the surface layer (shown below) and this integrated property is kept independent of $h$.

In an equlibrium state, the total chemical potential $\mu=\mu_{\mathrm{CH}}+\partial \bar{\gamma}_{\mathrm{FS}} / \partial \phi$ is spatially invariant and the diffusive current density $\boldsymbol{J}$ in equation (2.17) vanishes. The various contributions to $\mu$, however, do vary spatially (across the surface layer) even if the system is in equilibrium. This is obvious since $\bar{\gamma}_{\mathrm{FS}}$ is localized to the surface layer. In order to be consistent with the scaling of $\bar{\gamma}_{\mathrm{FS}} \sim 1 / h$ in equation (3.2), the $\mathrm{CH}$ chemical potential $\mu_{\mathrm{CH}}, \partial \bar{\gamma}_{\mathrm{FS}} / \partial \phi$, and the total chemical potential $\mu=\mu_{\mathrm{CH}}+\partial \bar{\gamma}_{\mathrm{FS}} / \partial \phi$ should all scale as $1 / h$, regardless of whether the system is in equilibrium or not. As a consequence, the surface-layer-integrated total chemical potential, $\int_{0}^{h} \mathrm{~d} z \mu$, is independent of $h$, i.e.

$$
\int_{0}^{h} \mathrm{~d} z \mu \sim \text { constant. }
$$

It will be shown later that the scaling $\mu_{\mathrm{CH}} \sim 1 / h$ determines the scaling of $\phi$ and the $h$-independent $\int_{0}^{h} \mathrm{~d} z \mu$ enters into the hydrodynamic boundary conditions.

Above the surface layer, the chemical potential in the bulk fluid is independent of $h$ (since we require the bulk hydrodynamics to stay the same to the leading order of $h$ ) and only varies slowly in space when the system is out of equilibrium. Furthermore, as far as the surface layer is concerned, the boundary value (at $z=h$ ) of the bulk chemical potential is small when compared to the $1 / h$ magnitude of the chemical potential within the surface layer. Thus to the leading order the boundary condition for the surface layer chemical potential at $z=h$ is effectively $\mu=0$. It follows that at equilibrium, the spatially invariant chemical potential is given by $\mu=0$ everywhere, from the surface layer to the bulk, although both $\mu_{\mathrm{CH}}$ and $\partial \bar{\gamma}_{\mathrm{FS}} / \partial \phi$ vary across the surface layer with a characteristic magnitude $\sim 1 / h$ (compared to which $\mu=0$ to the leading order).

When the system is out of equilibrium, the chemical potential $\mu$ varies across the surface layer with a charactersitic magnitude $\sim 1 / h$ (with $\int_{0}^{h} \mathrm{~d} z \mu$ being independent of $h$ ), subject to the boundary condition $\left.\mu\right|_{z=h}=0$ to the leading order. Physically, the spatial variations in $\mu$ (and consequently the non-zero diffusive transport measured by $\boldsymbol{J}=-M \nabla \mu)$ can be regarded as a manifestation of the Onsager principle of minimum energy dissipation when the system is driven away from equilibrium by, say, the shear flow imposed by the solid boundary: Given the presence of $\Phi_{d}=\frac{1}{2} \int \mathrm{d} \boldsymbol{r}\left[\boldsymbol{J}^{2} / M\right]$ in the action functional $A$ in equation (2.14), minimization of $A$ requires the participation of diffusive transport, localized to the fluid-fluid interfacial region. The overall dissipation will be reduced by introducing the two-phase diffusion into the viscous flow. This effect is particularly evident when the mobility coefficient $M$ is large enough (Qian et al. 2006). It follows that in the dynamic non-equilibrium two-phase flow, the chemical potential in the surface layer is distinct from the rest of the system, with a fast spatial variation along the $z$-direction (to be shown in figure 5 below). And since the diffusive transport is significant only in the region of diffuse fluid-fluid interface, there is also a spatial variation of the chemical potential along the $x$-direction (noted in the caption to figure 5). In summary, we conclude that the spatially varying chemical potential is localized to a region in the vicinity of the moving contact line. 
Another way of stating the above scaling relations is that $\int_{0}^{h} \mathrm{~d} z \bar{\beta}, \int_{0}^{h} \mathrm{~d} z \bar{\gamma}_{\mathrm{FS}}$, and $\int_{0}^{h} \mathrm{~d} z \mu$ are all $h$-independent constants which would appear in or affect the boundary conditions.

\subsubsection{Scaling of $\phi$ and $\boldsymbol{v}$}

To have $\mu_{\mathrm{CH}}=3 \gamma /(2 \sqrt{2})\left[-\xi \nabla^{2} \phi+(1 / \xi) \phi\left(\phi^{2}-1\right)\right] \sim 1 / h, \phi$ has to scale as

$$
\phi(z)=\phi^{(0)}+h \phi^{(1)}(z / h)+O\left(h^{2}\right) .
$$

From the natural boundary condition $\left.\partial_{z} \phi\right|_{z=0}=0$ and $\partial_{z}^{2} \phi \sim 1 / h$, it follows that the accumulated change of $\phi$ across the surface layer is on the order of $h^{2} \partial_{z}^{2} \phi \sim h$, a negligible quantity. It follows that $\phi$ is nearly constant in the surface layer. In addition, it is noted that the scaling $\left.\partial_{z} \phi\right|_{z=h} \sim h \partial_{z}^{2} \phi \sim$ const. on top of the surface layer is consistent with the requirement that the overall effect of the surface layer on the bulk be $h$-independent.

We further propose that the fluid velocity $v$ scales as

$$
\boldsymbol{v}(z)=\boldsymbol{v}^{(0)}+h \boldsymbol{v}^{(1)}(z / h)+O\left(h^{2}\right) .
$$

This ensures $v_{x}$ to be nearly constant across the surface layer so that the area density of the frictional force due to the wall, $\beta v_{x}$, is $h$-independent. It also leads to the scaling $\left.\partial_{z} v_{x}\right|_{z=h} \sim$ const. on top of the surface layer, a condition necessitated by the requirement that the shear rate in the bulk immediately above the surface layer be $h$-independent.

From the above, we conclude that $\phi, \partial_{z} \phi, v$ and $\partial_{z} v_{x}$ in the surface layer are directly related to those appearing in the boundary conditions.

\subsubsection{Scaling of $\eta$ and $M$}

The scaling for the viscosity $\eta=\eta(z ; \phi)$ may be deduced from the tangential $(x)$ component of the Stokes equation in which the body force densities $\mu \partial_{x} \phi$ and $-\bar{\beta} v_{x}$ both scale as $1 / h$ so that the corresponding forces per unit area are $h$ independent. Naturally, the tangential viscous force density $\nabla \cdot \sigma^{v} \cdot \hat{x}$ in the same equation, dominated by $\partial_{z}\left(\eta \partial_{z} v_{x}\right)$, has to scale as $1 / h$ so that the shear viscous stress at $z=h,\left.\eta \partial_{z} v_{x}\right|_{z=h}=\left.\eta \partial_{z} v_{x}\right|_{z=0}+\int_{0}^{h} \mathrm{~d} z\left[\partial_{z}\left(\eta \partial_{z} v_{x}\right)\right]$, is $h$-independent (with the natural boundary $\left.\eta \partial_{z} v_{x}\right|_{z=0}=0$ ). Combining this requirement with the scaling of $v_{x}$, we find the viscosity to scale as $\eta=\eta(z / h ; \phi)$.

To deduce the scaling for the $M=M(z ; \phi)$, we note that in the surface layer, the diffusion equation has the form $\dot{\phi}=\nabla \cdot[M \nabla \mu]$. Since the leading-order contribution to $\dot{\phi} \sim \partial \phi^{(0)} / \partial t+v_{x}^{(0)} \partial_{x} \phi^{(0)}$ is independent of $h$, it follows that the right-hand side of the equation must also scale in the same manner. Given the surface layer thickness as the smallest length scale, the tangential variation of the chemical potential along the surface can be neglected. Consequently, the diffusive current is dominated by its normal component $J_{z}=-M \partial_{z} \mu$, and the diffusion equation becomes $\dot{\phi}=\partial_{z}\left[M \partial_{z} \mu\right]$ in which each $\partial_{z}$ contributes a factor $1 / h$ and $\mu$ scales as $1 / h$. Together they require the mobility coefficient to scale as $M=h^{3} m(z / h ; \phi)$, where $m$ denotes an $h$-independent function.

Thus $\eta$ and $M / h^{3}$ should be proportional to the parameters that would appear in the boundary conditions.

\subsubsection{A summary}

From the above it follows that the normal diffusive current $J_{z}=-M \partial_{z} \mu$ should scale as $h$. This is consistent with the solution of the continuity equation $\dot{\phi}=-\partial_{z} J_{z}$, 


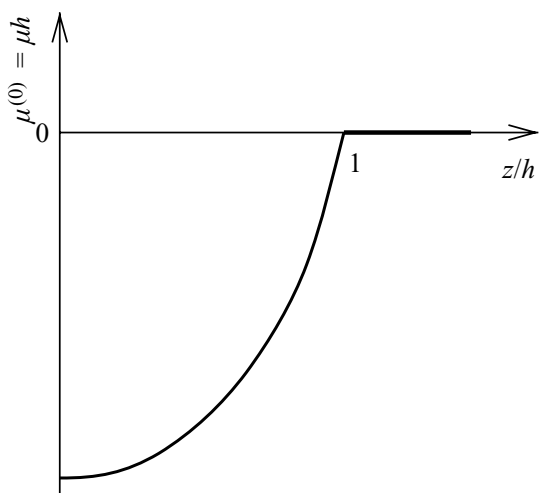

FIGURE 5. A schematic illustration for the variation of the chemical potential $\mu$ as a function of $z$ in the surface layer $(0<z<h)$, with the boundary conditions $\left.\partial_{z} \mu\right|_{z=0}=0$ and $\left.\mu\right|_{z=h}=0$. For constant $M=h^{3} m$ independent of $z$, integrating $\mu=\int_{h}^{z} \mathrm{~d} z^{\prime}\left[\dot{\phi} z^{\prime} / M\left(z^{\prime}\right)\right]$ yields $\mu^{(0)}=\mu h=(\dot{\phi} / 2 m)\left[(z / h)^{2}-1\right]$ as the scaled chemical potential which scales as $h^{0}$. The bulk chemical potential is nearly a constant. Hence multiplication with $h$ makes it very small, as indicated by the flat portion of the solid line. We note that the magnitude of $\mu^{(0)}$ is proportional to $\dot{\phi}$. Since $\dot{\phi}$ is non-zero only in the fluid-fluid interfacial region, it follows that $\mu^{(0)}$ exhibits the depicted behaviour only in the vicinity of the moving contact line.

$J_{z}=-\dot{\phi} z$, with the natural boundary condition $\left.J_{z}\right|_{z=0}=0$. From $-M \partial_{z} \mu=-\dot{\phi} z$, we can express the total chemical potential in the form $\mu=\int_{h}^{z} \mathrm{~d} z^{\prime}\left[\dot{\phi} z^{\prime} / M\right]$, with the boundary condition $\left.\mu\right|_{z=h}=0$. The salient behaviour of the chemical potential is illustrated in figure 5.

In summary, through physical considerations we have the following scaling relations for the parameters/variables in the surface layer:

$$
\begin{gathered}
\bar{\beta}=\frac{1}{h} D_{\beta}(\zeta ; \phi) \beta(\phi), \\
\bar{\gamma}_{\mathrm{FS}}=\frac{1}{h} D_{\gamma}(\zeta ; \phi) \gamma_{\mathrm{FS}}(\phi), \\
\eta=\eta(\zeta ; \phi), \\
M=h^{3} m(\zeta ; \phi), \\
\phi(z)=\phi^{(0)}+h \phi^{(1)}(\zeta)+O\left(h^{2}\right), \\
\boldsymbol{v}(z)=\boldsymbol{v}^{(0)}+h \boldsymbol{v}^{(1)}(\zeta)+O\left(h^{2}\right), \\
J_{z}=h(-\dot{\phi} \zeta), \\
\mu(z)=\mu^{(0)}(\zeta) / h,
\end{gathered}
$$

with $\mu^{(0)}(1)=0$. Here $\zeta=z / h$ is the scaled coordinate and the functions $D_{\beta}(\zeta ; \phi)$, $D_{\gamma}(\zeta ; \phi), \eta(\zeta ; \phi)$, and $m(\zeta ; \phi)$ are functions independent of $h$, with $h$-independent $\left.\partial_{z} \phi\right|_{z=h}$ and $\left.\partial_{z} v_{x}\right|_{z=h}$.

\subsection{Emergence of boundary conditions in variational form}

With the physically motivated scaling relations, the Onsager functional $A=\Phi+\dot{F}$ can be reduced to a form that takes into account only the integrated effects of the 
surface layer. And from this reduced variational functional the boundary conditions emerge as the result of functional minimization.

In equation (2.14), there are three terms in $\Phi=\Phi_{v}+\Phi_{s}+\Phi_{d}$ and two terms in $\dot{F}$ associated with $F_{\mathrm{CH}}$ and $F_{\mathrm{FS}}$. The idea here is to divide all the volume integrals into two regions, one extending from $z=0$ to $h$, the surface layer, and another from $z=h$ and beyond, denoted the bulk, as illustrated in figure 1. Below we first present the surface layer part.

Using the scaling relations in equations (3.6) to (3.13), we can obtain the reduced action functional $A^{\mathrm{SL}}$ :

$$
A^{\mathrm{SL}}[\boldsymbol{v}(\boldsymbol{r}), \dot{\phi}(\boldsymbol{r})]=\int \mathrm{d} S\left[\frac{\beta}{2} v_{x}^{2}\right]+\int \mathrm{d} S\left[\frac{\dot{\phi}^{2}}{2 \Gamma}\right]+\int \mathrm{d} S\left[L\left(\dot{\phi}-v_{x} \partial_{x} \phi\right)\right],
$$

for the surface layer to the leading order of $h^{0}$, with

$$
\frac{1}{\Gamma}=\int_{0}^{h} \mathrm{~d} z\left[\frac{z^{2}}{M}\right]=\int_{0}^{1} \mathrm{~d} \zeta\left[\frac{\zeta^{2}}{m}\right]
$$

and

$$
L=-\frac{3 \gamma \xi}{2 \sqrt{2}} \partial_{z} \phi+\frac{\partial \gamma_{\mathrm{FS}}}{\partial \phi} .
$$

The details involved in deriving equations (3.14), (3.15), and (3.16) are presented in Appendix C. Here we have used $\partial \phi / \partial t=\dot{\phi}-v_{x} \partial_{x} \phi$ for the surface layer. The surface integral is understood to be at $z=h$, distinct from that in equation (2.14). This point should be obvious, since by integrating $z$ from 0 to $h$, the effect of the surface layer on the bulk is now completely transmitted at their interface.

For the bulk part $(z>h)$, it is simply given by

$$
\begin{aligned}
& A^{\text {Bulk }}[\boldsymbol{v}(\boldsymbol{r}), \boldsymbol{J}(\boldsymbol{r})]=\int \mathrm{d} \boldsymbol{r}\left[\frac{\eta}{4}\left(\partial_{i} v_{j}+\partial_{j} v_{i}\right)^{2}\right]+\int \mathrm{d} \boldsymbol{r}\left[\frac{\boldsymbol{J}^{2}}{2 M}\right] \\
&+\int \mathrm{d} \boldsymbol{r}\left[\nabla \mu_{\mathrm{CH}} \cdot \boldsymbol{J}-\mu_{\mathrm{CH}}(\boldsymbol{v} \cdot \nabla \phi)\right],
\end{aligned}
$$

as can be deduced from equation (2.14) after the surface layer part is subtracted. The boundary conditions may be deduced by minimizing $A^{\mathrm{SL}}+A^{\text {Bulk }}$ with respect to $v_{x}$ and $\dot{\phi}$ (since minimization with respect to $\boldsymbol{J}$ leads only to a bulk equation of motion) at the surface $z=h$. Remembering that the bulk viscous term in equation (3.17) also contributes a surface term (tangential viscous stress), we obtain

$$
\begin{gathered}
\dot{\phi}=-\Gamma L, \\
\beta v_{x}=\eta \partial_{z} v_{x}+L \partial_{x} \phi .
\end{gathered}
$$

Owing to the scaling relations, equations (3.18) and (3.19) remain invariant when we take the $h \rightarrow 0^{+}$limit. Thus they are precisely the GNBCs (Qian et al. 2003, 2006). Note that we have been working in a reference frame where the solid surface (in the $x, y$-plane) is still and hence $v_{x}$ in equation (3.19) is identical to the tangential slip velocity. In the reference frame where the solid wall is moving along the $x$-direction with velocity $V_{w}, v_{x}$ should be replaced by the slip velocity $v_{x}^{\text {slip }}=v_{x}-V_{w}$.

To relate equations (3.18) and (3.19) to the dynamic contact angle $\theta_{d}$ defined at $z=h \rightarrow 0^{+}$(illustrated in figure 3 ), we consider a gently curved interface with a straight contact line parallel to the $y$-axis. Integrating $L \partial_{x} \phi$ along the $x$-axis across the 
fluid-fluid interface yields $\int_{\text {int }} \mathrm{d} x\left[L \partial_{x} \phi\right]=\gamma \cos \theta_{d}+\gamma_{2}-\gamma_{1}$, with $\gamma_{1}=\gamma_{\mathrm{FS}}(\phi=-1)$ and $\gamma_{2}=\gamma_{\mathrm{FS}}(\phi=+1)$ (see figure 3 ). At equilibrium $L=0$, from which we obtain $\int_{i n t} \mathrm{~d} x\left[L \partial_{x} \phi\right]=\gamma \cos \theta_{s}+\gamma_{2}-\gamma_{1}=0$ (the Young equation), in which the dynamic contact angle $\theta_{d}$ is replaced by the static contact angle $\theta_{s}$, also defined at $z=h \rightarrow 0^{+}$. It follows that $\int_{i n t} \mathrm{~d} x\left[L \partial_{x} \phi\right]=\gamma\left(\cos \theta_{d}-\cos \theta_{s}\right)$; hence $L \partial_{x} \phi$ is the uncompensated Young stress which originates from the deviation of the fluid-fluid interface from its static configuration. It should be noted that the uncompensated Young stress is spatially peaked in the vicinity of the contact line, so that away from the contact line $L=0$ and the GNBCs reduce to the Navier boundary condition $\beta v_{x}=\eta \partial_{z} v_{x}$, i.e. the slip velocity is proportional to the tangential viscous stress (Navier 1823).

\subsection{Boundary conditions as the mathematical consequence of scaling the equations of motion}

Whereas in $\S 3.1$ and $\S 3.2$ the surface-layer thickness $h$ is treated as a small but finite quantity, here we carry out the mathematical limiting process of $h \rightarrow 0^{+}$by using the scaling relations, (3.6) to (3.13), to examine the resulting surface layer equations of motion, (2.16) and (2.18), in that limit. Besides offering a consistency check, this approach offers a more precise context in which the boundary conditions emerge, as well as a framework to delineate the limitations on the validity of the boundary conditions, e.g. by examining the higher-order terms in the limiting process.

Substituting the scaling relations (3.6), (3.7), (3.8), (3.10), and (3.11) into the $x$ component of the Stokes equation,

$$
\begin{aligned}
& -\partial_{x} p+\partial_{x}\left[2 \eta\left(\partial_{x} v_{x}\right)\right]+\partial_{z}\left[\eta\left(\partial_{z} v_{x}+\partial_{x} v_{z}\right)\right] \\
& \quad+\left\{\frac{3 \gamma}{2 \sqrt{2}}\left[-\xi\left(\partial_{x}^{2} \phi+\partial_{z}^{2} \phi\right)+\frac{1}{\xi} \phi\left(\phi^{2}-1\right)\right]+\frac{\partial \bar{\gamma}_{\mathrm{FS}}}{\partial \phi}\right\} \partial_{x} \phi-\bar{\beta} v_{x}=0,
\end{aligned}
$$

yields to the leading order of $h^{-1}$

$$
\begin{aligned}
\frac{1}{h} \partial_{\zeta}\left[\eta\left(\zeta ; \phi^{(0)}\right) \partial_{\zeta} v_{x}^{(1)}\right]-\frac{1}{h} \frac{3 \gamma \xi}{2 \sqrt{2}} \partial_{\zeta}^{2} \phi^{(1)} \partial_{x} \phi^{(0)} & +\left.\frac{1}{h} \frac{\partial}{\partial \phi}\left[D_{\gamma}(\zeta ; \phi) \gamma_{\mathrm{FS}}(\phi)\right]\right|_{\phi=\phi^{(0)}} \partial_{x} \phi^{(0)} \\
& -\frac{1}{h} D_{\beta}\left(\zeta ; \phi^{(0)}\right) \beta\left(\phi^{(0)}\right) v_{x}^{(0)}=0, \quad
\end{aligned}
$$

where $\zeta=z / h$. With the help of the natural boundary conditions $\partial_{\zeta} \phi^{(1)}=0$ and $\partial_{\zeta} v_{x}^{(1)}=0$ at $\zeta=0$, integrating the above equation from $\zeta=0$ to 1 yields

$$
\begin{array}{r}
{\left.\left[\eta\left(\zeta ; \phi^{(0)}\right) \partial_{\zeta} v_{x}^{(1)}\right]\right|_{\zeta=1}+\left\{-\left.\frac{3 \gamma \xi}{2 \sqrt{2}}\left[\partial_{\zeta} \phi^{(1)}\right]\right|_{\zeta=1}+\left.\frac{\partial \gamma_{\mathrm{FS}}(\phi)}{\partial \phi}\right|_{\phi=\phi^{(0)}}\right\} \partial_{x} \phi^{(0)}} \\
-\beta\left(\phi^{(0)}\right) v_{x}^{(0)}=0 .
\end{array}
$$

In the $h \rightarrow 0^{+}$limit, equation (3.22) becomes the hydrodynamic boundary condition, equation (3.19) at $z=0^{+}$. This boundary condition governs the tangential momentum transport across the fluid-solid interface.

The other hydrodynamic boundary condition can be derived by using the scaling solution for the advection-diffusion equation

$$
\dot{\phi}=\nabla \cdot\left[M \nabla\left(\mu_{\mathrm{CH}}+\frac{\partial \bar{\gamma}_{\mathrm{FS}}}{\partial \phi}\right)\right] .
$$


It can be shown that, to the leading order of $h^{0}$, the material derivative $\dot{\phi}$ in the surface layer can be expressed as

$$
\dot{\phi}=-\Gamma\left\{-\left.\frac{3 \gamma \xi}{2 \sqrt{2}}\left[\partial_{\zeta} \phi^{(1)}\right]\right|_{\zeta=1}+\left.\frac{\partial \gamma_{\mathrm{FS}}(\phi)}{\partial \phi}\right|_{\phi=\phi^{(0)}}\right\},
$$

where

$$
\frac{1}{\Gamma}=-\int_{0}^{1} \mathrm{~d} \zeta\left\{\int_{1}^{\zeta} \mathrm{d} \zeta^{\prime}\left[\frac{\zeta^{\prime}}{m\left(\zeta^{\prime} ; \phi^{(0)}\right)}\right]\right\} .
$$

The details involved in deriving equations (3.24) and (3.25) are presented in Appendix D. In the $h \rightarrow 0^{+}$limit, equation (3.24) yields the hydrodynamic boundary condition, equation (3.18) at $z=0^{+}$. Thus together with equation (3.22), we have recovered the GNBCs through an alternative route. It is interesting to note that, for constant $m\left(\zeta ; \phi^{(0)}\right)=m$, an explicit relation between the mobility coefficient $M=h^{3} m$ and the relaxational rate coefficient $\Gamma$ can be derived:

$$
\frac{1}{\Gamma}=-\frac{1}{m} \int_{0}^{1} \mathrm{~d} \zeta\left[\int_{1}^{\zeta} \zeta^{\prime} \mathrm{d} \zeta^{\prime}\right]=\frac{h^{3}}{3 M} .
$$

An identical result can also be deduced from equation (3.15). It should be noted that in restoring $M$ (in place of $m$ ) on the right-hand side of equation (3.26), $h$ is the value of the original surface-layer thickness, before the limiting process is taken.

It follows from the above that if we let $h$ approach zero with the condition of invariant boundary conditions, then the parameters/variables in the surface layer must scale with $h$ in the manner specified by equations (3.6)-(3.13).

\subsection{Implications}

Three implications can be drawn from this section. First, consistency of the scaling relations demonstrates that hydrodynamic boundary conditions are a natural manifestation of the Onsager principle as it is applied to the surface layer. Second, since the parameters of the boundary conditions are directly tied to those in the surface layer, their values can be related to the coefficients in the bulk equations of motion, but under the influence of the wall potential (Barrat \& Bocquet 1999; Bocquet \& Barrat 2007). Third, since the boundary conditions are the limiting form of the equations of motion in the surface layer, they are part of the linear response phenomena underpinned by the Onsager variational principle. In particular, since the Stokes equation is also a consequence of the Onsager principle (see equation (2.16)), the use of the boundary conditions (3.18) and (3.19) in continuum hydrodynamics would guarantee (i) force balance and (ii) the predicted dynamics to be statistically the most probable course of dissipative processes. In this regard we should note that both boundary conditions, the relaxational (Allen-Cahn) equation for the phase field variable (3.18) and the linear friction law (3.19), have their counterparts in the bulk equations of motion. This is especially clear from their derivation by scaling the surface-layer equations of motion. Thus the fact that they can resolve the moving contact line problem (Dussan V. \& Davis 1974) should not be a surprise from two points of view. The first is that by requiring the continuum hydrodynamics to be consistent with the same statistical mechanical principle as that underlying molecular dynamics, the same (time-averaged) dynamic behaviour is expected. And since all molecular interactions are finite in their magnitudes, no dissipative divergence should arise. The second is that the Onsager principle inherently minimizes energy dissipation, hence dissipative divergence is always avoided. 

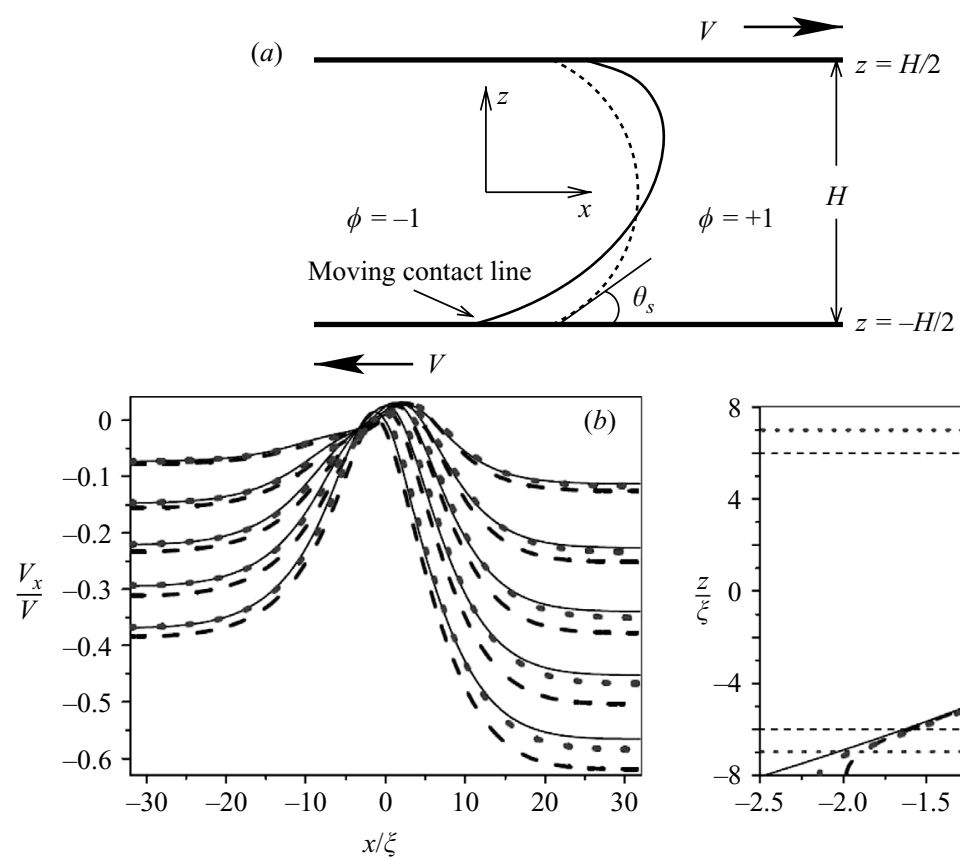

Figure 6. (a) A schematic illustration for the immiscible two-phase Couette flow confined between two planar solid surfaces that are parallel to the $(x, y)$-plane and separated by a distance $H$. The upper surface moves with velocity $V \hat{\boldsymbol{x}}$ and the lower surface moves with velocity $-V \hat{\boldsymbol{x}}$. Under the shear movement, the fluid-fluid interface (solid curve) deviates from its static configuration (dashed circular arc) which has static contact angle $\theta_{s}$. The channel is long enough along the $x$-direction to ensure that single-phase linear Couette flow is recovered far away from the fluid-fluid interface. $(b)$ The tangential velocity profiles $v_{x}(x)$ at different $z$ levels $(z / \xi=-20 / 3,-16 / 3,-12 / 3,-8 / 3,-4 / 3)$. The solid lines denote the results obtained by employing the boundary-condition description; the dashed and dotted lines denote the results obtained by employing the surface-layer description, for $h=2 \xi$ and $h=\xi$ respectively. (c) The interface profiles (the locus $\phi=0$ ). The curves have the same meaning as in $(b)$. The thin horizontal lines mark the interface between the surface layer and the bulk fluid, for $h=2 \xi$ (dashed lines) and $h=\xi$ (dotted lines) respectively.

\section{Numerical verification}

Numerical calculations have been carried out to demonstrate that the effects of the surface layer on the bulk can be approximately described by the hydrodynamic boundary conditions. Consider two immiscible fluids confined between two planar solid surfaces parallel to the (x,y)-plane (Qian et al. 2003, 2004, 2006). Couette flow is generated by moving the upper surface with velocity $V \hat{\boldsymbol{x}}$ and the lower surface with velocity $-V \hat{\boldsymbol{x}}$ (see figure $6 a$ ). From both MD simulations and continuum calculations based on the GNBCs, it has been shown that along the solid surface there is a smooth transition from near-complete slip at the moving contact line to near-zero partial slip far away from it (Qian et al. 2004). To show quantitatively the near equivalence of the surface-layer description and boundary-condition description, we have carried out two types of calculations. The first type employs the surface-layer description in which the effects of the solid wall are described by the Navier-Stokes equation (obtained by adding the inertia term to equation (2.16)) and the convection-diffusion equation (2.18) coupled with the natural boundary conditions (2.20), (2.21), and (2.22). The second type employs the GNBCs (3.18) and (3.19). 
Figures $6(b)$ and $6(c)$ show the tangential velocity profiles $\left(v_{x}(x)\right.$ at different $z$ levels) and the interface profiles (the locus $\phi=0$ ), obtained from the two types of calculations. The solid lines denote the results obtained by employing the boundary-condition description; the dashed and dotted lines denote the results obtained by employing the surface-layer description for two different values of $h$. The near equivalence of the two descriptions is quantitatively demonstrated. In particular, it is seen that beyond the surface layer, the calculated curves of the three cases tend to coincide. Deviations between the two descriptions, however, are clearly visible in figures $6(b)$ and $6(c)$. They are mostly due to the inaccuracy caused by using a slip length $l_{s}=\eta / \beta$ to describe a surface layer whose thickness $h$ is not sufficiently small compared to the slip length. This is evident in figure $6(b)$ : relatively larger deviations are found in the right half of the space associated with a smaller slip length. The agreement for the interface profiles is better than that for the velocity profiles. The three curves in figure 6(c) tend to coincide quickly in the bulk away from the surface layer. The overall agreement of the two descriptions is seen to be improved when a smaller surface-layer thickness $h=\xi$ is used. This is consistent with the mathematical limiting process of $h \rightarrow 0^{+}$in which the GNBCs emerge as leading-order effects of the surface-layer hydrodynamics on the bulk. We note that in reducing $h$, those parameters for the surface layer, e.g. $\bar{\beta}, \bar{\gamma}_{\mathrm{FS}}$, and $M$, all treated as $z$-independent quantities here, have to be scaled in order to keep the corresponding parameters $\left(\beta, \gamma_{\mathrm{FS}}\right.$, and $\left.\Gamma\right)$ in the GNBCs invariant. From these comparisons it follows that as the transverse $z$ dimension of the system increases relative to the surface-layer thickness $h$, the GNBCs accuracy improves monotonically so that in almost all practical cases the hydrodynamic boundary conditions might be treated as being exact.

The parameters used in the calculations are as follows. Taking $\xi$ as the length unit, $V_{0}$ as the velocity unit, and $\eta V_{0} / \xi$ as the stress unit, we carry out the GNBC calculations for the dimensionless control parameters $\theta_{s}=67.6^{\circ}, M \gamma / V_{0} \xi^{2}=0.47$, $\rho V_{0} \xi / \eta=0.003, \eta V_{0} / \gamma=0.088, \Gamma \gamma \xi / V_{0}=0.18, \eta / \beta_{1} \xi=10$ and $\eta / \beta_{2} \xi=3.8$ (where $\beta_{1}=\beta(\phi=-1)$ and $\left.\beta_{2}=\beta(\phi=+1)\right), H / \xi=16$, and $V / V_{0}=0.5$. As for the surfacelayer calculations, $\bar{\beta}=\beta / h, \bar{\gamma}_{\mathrm{FS}}=\gamma_{\mathrm{FS}} / h$, and $M=\Gamma h^{3} / 3$ in the surface layer are all $z$-independent quantities whose values scale with $h$ in order to keep the corresponding parameters $\beta, \gamma_{\mathrm{FS}}$, and $\Gamma$ in the GNBCs invariant as $h \rightarrow 0^{+}$.

It should be noted that the use of the GNBC has resolved the classical problem of the moving contact line (Qian et al. 2003, 2004, 2006), with the MD simulation results quantitatively reproduced by continuum hydrodynamics. This should be expected, since molecular dynamics must follow the statistical mechanical principle as embodied in the Onsager principle, as noted earlier. Below we demonstrate the application of the same approach to the derivation of liquid-crystal hydrodynamic boundary conditions.

\section{Liquid-crystal hydrodynamic boundary conditions}

Nematic liquid crystals (NLCs) have no translational long-range order and therefore flow like a fluid. However, they have long-range orientational order, which gives rise to many special properties, from orientational elasticity to anisotropic viscosity (de Gennes \& Prost 1993). In particular, optical birefriengence is the basis of NLCs widespread application in display devices. NLC hydrodynamics is composed of two elements: the orientational elastic free energy, developed by Oseen, Zocher, and Frank (Oseen 1933; Zocher 1933; Frank 1958); and dissipative stress tensors, developed by Ericksen, Leslie, and Parodi (Ericksen 1960; Leslie 1968; Parodi 1970). In the presence of long-range orientational order, the alignment state of the NLCs is locally 
characterized by a unit vector $\boldsymbol{n}$, the director, that denotes the axis of uniaxial ordering. In a state of elastic distortion described by a variable director $\boldsymbol{n}(\boldsymbol{r})$, the elastic distortion free energy is given by

$$
F_{d}[\boldsymbol{n}(\boldsymbol{r})]=\int \mathrm{d} \boldsymbol{r}\left[f_{d}(\boldsymbol{n}, \nabla \boldsymbol{n})\right],
$$

in which $f_{d}$ is the Frank-Oseen free energy density:

$$
f_{d}(\boldsymbol{n}, \nabla \boldsymbol{n})=\frac{1}{2} K_{1}(\nabla \cdot \boldsymbol{n})^{2}+\frac{1}{2} K_{2}(\boldsymbol{n} \cdot \nabla \times \boldsymbol{n})^{2}+\frac{1}{2} K_{3}(\boldsymbol{n} \times \nabla \times \boldsymbol{n})^{2},
$$

where $K_{1}, K_{2}$, and $K_{3}$ are the splay, twist, and bend elastic constants, respectively. The rate of change of the elastic energy can be written as

$$
\dot{F}_{d}=\int \mathrm{d} \boldsymbol{r}\left[\left(-\partial_{j} \pi_{j k}+\frac{\partial f_{d}}{\partial n_{k}}\right) \frac{\partial n_{k}}{\partial t}\right]+\int \mathrm{d} S\left[\boldsymbol{m}_{j} \pi_{j k} \frac{\partial n_{k}}{\partial t}\right],
$$

where $\pi_{j k}=\partial f_{d} / \partial\left(\partial_{j} n_{k}\right), \boldsymbol{m}$ is the outward pointing unit vector normal to the solid surface, and $\int \mathrm{d} S$ denotes the surface integral at the solid surface. The elastic free energy gives rise to the distortion stress tensor $\boldsymbol{\sigma}^{d}$, given by $\sigma_{j i}^{d}=-\pi_{j k} \partial_{i} n_{k}+f_{d} \delta_{j i}$, and the distortion molecular field $\boldsymbol{H}^{d}$, given by $H_{k}^{d}=\partial_{j} \pi_{j k}-\partial f_{d} / \partial n_{k}$ (de Gennes \& Prost 1993). It is easy to show that $\dot{F}_{d}$ can be written as

$$
\dot{F}_{d}=\int \mathrm{d} \boldsymbol{r}\left[-H_{k}^{d} \dot{n}_{k}\right]+\int \mathrm{d} S\left[\boldsymbol{m}_{j} \pi_{j k} \dot{n}_{k}\right]+\int \mathrm{d} \boldsymbol{r}\left[\sigma_{j i}^{d} \partial_{j} v_{i}\right],
$$

where $\dot{n}_{k}=\partial n_{k} / \partial t+v_{j} \partial_{j} n_{k}$ is the material derivative. Under the one-constant approximation $K=K_{1}=K_{2}=K_{3}$, the elastic energy density becomes $f_{d}=\frac{1}{2} K\left(\partial_{j} n_{k}\right)^{2}$, and consequently $\sigma_{j i}^{d}=-K \partial_{j} n_{k} \partial_{i} n_{k}+f_{d} \delta_{j i}$ and $H_{k}^{d}=K \nabla^{2} n_{k}$ (Yue et al. 2004).

For the dissipative part of the Onsager variational action, it is necessary to first define the viscous stress tensor $\boldsymbol{\sigma}^{v}$ and the frictional molecular field $\boldsymbol{H}^{v}$ :

$$
\begin{gathered}
\sigma_{\alpha \beta}^{v}=\alpha_{1} n_{\alpha} n_{\beta} n_{\mu} n_{\nu} e_{\mu \nu}+\alpha_{4} e_{\alpha \beta}+\alpha_{5} n_{\alpha} n_{\mu} e_{\mu \beta}+\alpha_{6} n_{\beta} n_{\mu} e_{\mu \alpha}+\alpha_{2} n_{\alpha} N_{\beta}+\alpha_{3} n_{\beta} N_{\alpha}, \\
-H_{\beta}^{v}=\left(\alpha_{3}-\alpha_{2}\right) N_{\beta}+\left(\alpha_{6}-\alpha_{5}\right) n_{\alpha} e_{\alpha \beta},
\end{gathered}
$$

where the six $\alpha_{i}$ are the Leslie viscosity coefficients, constrained by the Parodi relation $\alpha_{2}+\alpha_{3}=\alpha_{6}-\alpha_{5}, \boldsymbol{e}=\frac{1}{2}\left[\nabla \boldsymbol{v}+(\nabla \boldsymbol{v})^{T}\right]$ is the rate of strain tensor, and the vector $\boldsymbol{N}=\dot{\boldsymbol{n}}-\boldsymbol{\omega} \times \boldsymbol{n}$ represents the rate of change of the director with respect to the background fluid, with $\omega=\frac{1}{2} \nabla \times \boldsymbol{v}$ being the angular velocity associated with the rigid-body rotation of the fluid (de Gennes \& Prost 1993). To obtain the constitutive relations (5.4) and (5.5), each contribution to the entropy production is written as the product of a 'flux' and its conjugate 'force', and each force is expressed as a linear combination of the fluxes (linear dissipative response) (de Gennes \& Prost 1993). Hence the corresponding dissipation function can be written as

$$
\Phi_{v}=\frac{1}{2} \int \mathrm{d} \boldsymbol{r}\left[\sigma_{j i}^{v}(\boldsymbol{e}, \boldsymbol{N}) \partial_{j} v_{i}-H_{k}^{v}(\boldsymbol{e}, \boldsymbol{N}) \dot{n}_{k}\right]=\frac{1}{2} \int \mathrm{d} \boldsymbol{r}\left[\sigma_{j i}^{v s}(\boldsymbol{e}, \boldsymbol{N}) e_{j i}-H_{k}^{v}(\boldsymbol{e}, \boldsymbol{N}) N_{k}\right],
$$

in which $\sigma^{\nu s}(\boldsymbol{e}, N)$ stands for the symmetric part of $\sigma^{v}(\boldsymbol{e}, N)$. It should be noted that angular momentum conservation requires the antisymmetric part of $\sigma^{v}(\boldsymbol{e}, N)$ to be related to $\boldsymbol{H}^{v}(\boldsymbol{e}, \boldsymbol{N})$ through the relation $\varepsilon_{i j k} \sigma_{j k}^{v}=\varepsilon_{i j k} n_{j} H_{k}^{v}$, from which we have in the dissipation function $\sigma_{j i}^{v} \partial_{j} v_{i}-H_{k}^{v} \dot{n}_{k}=\sigma_{j i}^{v s} e_{j i}-H_{k}^{v} N_{k}$. Note that due to the Parodi relation, the matrix of the frictional coefficients in the quadratic functional $\Phi_{v}$ is 
symmetric, hence

$$
\delta\left\{\frac{1}{2}\left[\sigma_{j i}^{v s}(\boldsymbol{e}, \boldsymbol{N}) e_{j i}-H_{k}^{v}(\boldsymbol{e}, \boldsymbol{N}) N_{k}\right]\right\}=\sigma_{j i}^{v s}(\boldsymbol{e}, \boldsymbol{N}) \delta e_{j i}-H_{k}^{v}(\boldsymbol{e}, \boldsymbol{N}) \delta N_{k},
$$

which can be further written as $\sigma_{j i}^{v}(\boldsymbol{e}, \boldsymbol{N}) \partial_{j} \delta v_{i}-H_{k}^{v}(\boldsymbol{e}, \boldsymbol{N}) \delta \dot{n}_{k}$ by using $\varepsilon_{i j k} \sigma_{j k}^{v}=$ $\varepsilon_{i j k} n_{j} H_{k}^{v}$. It follows that the variation of $\Phi_{v}$ is of the form

$$
\delta \Phi_{v}=\int \mathrm{d} \boldsymbol{r}\left[\sigma_{j i}^{v}(\boldsymbol{e}, \boldsymbol{N}) \partial_{j} \delta v_{i}-H_{k}^{v}(\boldsymbol{e}, \boldsymbol{N}) \delta \dot{n}_{k}\right] .
$$

According to the Onsager principle, by minimizing the functional $A=\Phi_{v}+\dot{F}_{d}$ with respect to $\boldsymbol{v}$ and $\dot{\boldsymbol{n}}$ we obtain the equations of force balance in the absence of inertial effects:

$$
-\nabla p+\nabla \cdot \sigma^{d}+\nabla \cdot \sigma^{v}=0
$$

and

$$
\boldsymbol{H}^{d}+\boldsymbol{H}^{v}-\lambda \boldsymbol{n}=0
$$

Here $\lambda$ is a Lagrange multiplier imposed by the constraint $\boldsymbol{n}^{2}=1$, and $p$ is similarly identified as the Lagrange multiplier imposed by the incompressibility condition (see equations (2.15) and (2.16)). Equations of force balance at the solid surface can also be derived from the Onsager principle. This leads to the natural boundary conditions, to be considered below.

\subsection{Surface-layer dynamics and scaling}

While bulk NLC has uniaxial symmetry, under the influence of wall potential the surface layer is in general a biaxial environment in which there can be two axes of symmetry - normal and parallel to the solid surface. This can happen, for example, when the wall potential defines a preferred orientational direction for $\boldsymbol{n}$ that has a component parallel to the surface. Thus the most general form of surface-layer hydrodynamics can be extremely complicated. For the purpose of demonstrating the general approach of deriving the boundary conditions, however, here we choose the simplest model of a uniaxial surface layer, i.e. the preferred direction for $\boldsymbol{n}$ coincides with the surface normal.

\subsubsection{Dissipation function}

The dissipation function pertaining to the surface layer consists of three terms:

$$
\begin{gathered}
\Phi_{s}=\frac{1}{2} \int \mathrm{d} \boldsymbol{r}\left[\bar{\beta} \boldsymbol{v}_{\tau}^{2}\right], \\
\Phi_{r}=\frac{1}{2} \int \mathrm{d} \boldsymbol{r}\left[\bar{\kappa} \dot{\boldsymbol{n}}^{2}\right], \\
\Phi_{c}=\int \mathrm{d} \boldsymbol{r}\left[\bar{\chi}(\boldsymbol{n} \cdot \boldsymbol{m})\left(\boldsymbol{v}_{\tau} \cdot \dot{\boldsymbol{n}}\right)\right],
\end{gathered}
$$

where $\Phi_{s}$ arises from the translational slip relative to the solid wall, $\Phi_{r}$ from the director rotation, and $\Phi_{c}$ from the cross-coupling between the translational and rotational processes. Here $\boldsymbol{v}_{\tau}=\boldsymbol{v} \cdot(\mathbf{1}-\boldsymbol{m} \boldsymbol{m})$ denotes the projection of $\boldsymbol{v}$ on the solid surface, $\bar{\beta}$ is the slip coefficient associated with the translational wall friction, $\bar{\kappa}$ is the coefficient associated with the rotational wall friction, and $\bar{\chi}$ is the coefficient that measures how strongly the translational and rotational processes interfere with each other. Assuming that the solid surface is in the $(x, y)$-plane and the NLC is in the half-space $z \geqslant 0$, we have $\boldsymbol{m}=-\hat{z}$, and $\bar{\beta}, \bar{\kappa}$, and $\bar{\chi}$ as functions of $z$, all approaching zero when $z>h$. The simple forms adopted for $\Phi_{s}, \Phi_{r}$, and $\Phi_{c}$ need a few remarks: 
(a) The dissipation function is quadratic in the rates. With the slip and rotation measured respectively by $\boldsymbol{v}_{\tau}$ and $\dot{\boldsymbol{n}}$, we have two diagonal terms, $\bar{\beta}(z) \boldsymbol{v}_{\tau}^{2}\left(\Phi_{s}\right)$ for the dissipation density due to slipping, and $\bar{\kappa}(z) \dot{\boldsymbol{n}}^{2}\left(\Phi_{r}\right)$ due to rotation. $\Phi_{c}$ is the offdiagonal cross-term which couples $\boldsymbol{v}_{\tau}$ and $\dot{\boldsymbol{n}}$. It should be noted that in general, terms like $(\boldsymbol{n} \cdot \boldsymbol{m})^{2} \boldsymbol{v}_{\tau}^{2}$ and $\left(\boldsymbol{n} \cdot \boldsymbol{v}_{\tau}\right)^{2}$ with $z$-dependent coefficients may also appear in $\Phi_{s}$, and similarly $(\boldsymbol{n} \cdot \boldsymbol{m})^{2} \dot{\boldsymbol{n}}^{2}$ and $(\boldsymbol{m} \cdot \dot{\boldsymbol{n}})^{2}$ with $z$-dependent coefficients may appear in $\Phi_{r}$. They do not affect the derivation of the boundary condition, and hence are neglected for simplicity. Below we let $\bar{\beta}$ and $\bar{\kappa}$ be locally dependent on $\boldsymbol{n}$, i.e. $\bar{\beta}=\bar{\beta}(z ; \boldsymbol{n}), \bar{\kappa}=\bar{\kappa}(z ; \boldsymbol{n})$.

(b) It is noted that the factor $\boldsymbol{n} \cdot \boldsymbol{m}$ is singled out in $\Phi_{c}$ for its dependence on $\boldsymbol{n}$, thereby making it simple to establish the correspondence with the similar term in the bulk dissipation. For $\alpha_{3}=0$, that term in the bulk comes from the cross-term in $-\alpha_{2}\left(N_{\beta}-n_{\alpha} e_{\alpha \beta}\right)^{2}=-\alpha_{2}\left(\dot{n}_{\beta}-n_{\alpha} \partial_{\alpha} v_{\beta}\right)^{2}$ with $\alpha_{2}<0$, i.e. $2 \alpha_{2} \dot{n}_{\beta} n_{\alpha} \partial_{\alpha} v_{\beta}$. It follows that for the surface layer $\bar{\chi}(\boldsymbol{n} \cdot \boldsymbol{m})\left(\boldsymbol{v}_{\tau} \cdot \dot{\boldsymbol{n}}\right)=\bar{\chi} \dot{n}_{\beta} n_{\alpha} m_{\alpha}\left[v_{\tau}\right]_{\beta}$ with $\bar{\chi}>0$ should be the corresponding form. This is based on the consideration that $\boldsymbol{v}_{\tau}$ is the velocity change from the solid to the surface layer, which takes place along the $-\boldsymbol{m}$ direction over a distance $\sim h$, and hence the role of $\partial_{\alpha} v_{\beta}$ in the bulk becomes $-h^{-1} m_{\alpha}\left[v_{\tau}\right]_{\beta}$ in the surface layer, and one can heuristically identify $\bar{\chi}=-2 \alpha_{2} / h$, where $\alpha_{2}$ should be the viscosity value in the surface layer, not necessarily the same as that in the bulk.

\subsubsection{Orientational alignment energy}

The orientational alignment energy distributed in the surface layer can be written in the Rapini-Papolar form (Rapini \& Papoular 1969) as $F_{w}=\int \mathrm{d} r\left[f_{w}(z ; \boldsymbol{n})\right]$, with an energy density

$$
f_{w}(z ; \boldsymbol{n})=-\frac{1}{2} \bar{w}(z)(\boldsymbol{n} \cdot \boldsymbol{m})^{2},
$$

in which $\bar{w}(z)$ is the distributed alignment strength that varies with $z$ and extends from 0 to $h$.

\subsubsection{Variational functional and Euler-Lagrange equations}

According to the Onsager principle, the surface-layer dynamics is governed by the variational functional $A=\Phi_{v}+\Phi_{s}+\Phi_{r}+\Phi_{c}+\dot{F}_{d}+\dot{F}_{w}$, which is to be minimized with respect to the rates $\{\boldsymbol{v}, \dot{\boldsymbol{n}}\}$, supplemented with the incompressibility condition. This leads to the equations for force balance:

$$
-\nabla p+\nabla \cdot \boldsymbol{\sigma}^{d}+\nabla \cdot \boldsymbol{\sigma}^{v}+\boldsymbol{g}^{w}+\boldsymbol{g}^{s}+\boldsymbol{g}^{c}=0,
$$

and

$$
\boldsymbol{H}^{d}+\boldsymbol{H}^{v}+\boldsymbol{H}^{w}+\boldsymbol{H}^{r}+\boldsymbol{H}^{c}-\lambda \boldsymbol{n}=0,
$$

where $\boldsymbol{g}^{w}, \boldsymbol{g}^{s}$, and $\boldsymbol{g}^{c}$ are the force densities, and $\boldsymbol{H}^{w}, \boldsymbol{H}^{r}$, and $\boldsymbol{H}^{c}$ the molecular fields, all narrowly distributed in the surface layer, with the superscripts $w, s, r$, and $c$ denoting their origins from $\dot{F}_{w}, \Phi_{s}, \Phi_{r}$, and $\Phi_{c}$, respectively, and $\lambda$ is a Lagrange multiplier imposed by $\boldsymbol{n}^{2}=1$. Explicit expressions for these surface-layer forcing terms are as follows:

$$
\begin{gathered}
\boldsymbol{g}^{w}=-\frac{1}{2} \nabla\left[\bar{w}(\boldsymbol{n} \cdot \boldsymbol{m})^{2}\right], \\
\boldsymbol{g}^{s}=-\bar{\beta} \boldsymbol{v} \cdot(\mathbf{1}-\boldsymbol{m} \boldsymbol{m}), \\
\boldsymbol{g}^{c}=-\bar{\chi}(\boldsymbol{n} \cdot \boldsymbol{m}) \dot{\boldsymbol{n}} \cdot(\mathbf{1}-\boldsymbol{m} \boldsymbol{m}), \\
\boldsymbol{H}^{w}=\bar{w}(\boldsymbol{n} \cdot \boldsymbol{m}) \boldsymbol{m},
\end{gathered}
$$



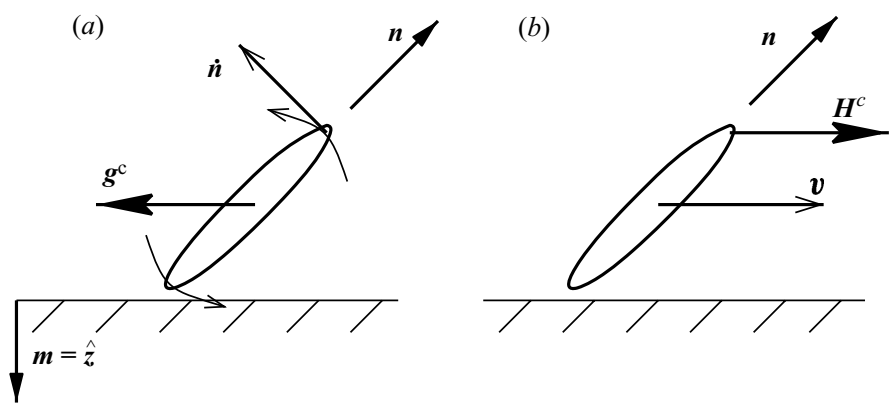

FIgURE 7. A schematic illustration for the two cross-coupling terms in the LC hydrodynamic boundary conditions: (a) the tangential frictional force $\boldsymbol{g}^{c}=-\bar{\chi}(\boldsymbol{n} \cdot \boldsymbol{m}) \dot{\boldsymbol{n}} \cdot(\mathbf{1}-\boldsymbol{m} \boldsymbol{m})$ due to director rotation measured by $\dot{\boldsymbol{n}}$, and $(b)$ the tangential molecular (rotational) field $\boldsymbol{H}^{c}=-\bar{\chi}(\boldsymbol{n} \cdot \boldsymbol{m}) \boldsymbol{v} \cdot(\mathbf{1}-\boldsymbol{m} \boldsymbol{m})$ induced by relative slip velocity, measured by $\boldsymbol{v}_{\tau}=\boldsymbol{v} \cdot(\mathbf{1}-\boldsymbol{m} \boldsymbol{m})$.

$$
\begin{gathered}
\boldsymbol{H}^{r}=-\bar{\kappa} \dot{\boldsymbol{n}}, \\
\boldsymbol{H}^{c}=-\bar{\chi}(\boldsymbol{n} \cdot \boldsymbol{m}) \boldsymbol{v} \cdot(\mathbf{1}-\boldsymbol{m} \boldsymbol{m}) .
\end{gathered}
$$

The frictional force $\boldsymbol{g}^{c}$ due to director rotation and the molecular field $\boldsymbol{H}^{c}$ due to slip represent the dynamic effects of the cross-term $\Phi_{c}$, as illustrated in figure 7 .

The natural boundary conditions

$$
\hat{z} \cdot\left(\sigma^{d}+\sigma^{v}\right) \cdot(1-\hat{z} \hat{z})=0
$$

and

$$
\hat{z} \cdot \pi-\lambda_{0} n=0
$$

are obtained by minimizing the action functional $A$ with respect to $v$ and $\dot{\boldsymbol{n}}$ at the surface $z=0$, with $\lambda_{0}$ a Lagrange multiplier imposed by $\boldsymbol{n}^{2}=1$. Note that substituting the second condition into the first one yields $\hat{z} \cdot \boldsymbol{\sigma}^{d} \cdot(\mathbf{1}-\hat{z} \hat{z})=0$, and hence $\hat{z} \cdot \boldsymbol{\sigma}^{v} \cdot(\mathbf{1}-$ $\hat{z} \hat{z})=0$ as well. Here and below we use the inward pointing unit vector $\hat{z}$ for all expressions.

\subsubsection{Scaling relations}

A set of scaling relations can be obtained by first requiring a few $z$-integrated properties that owe their origin to the wall be independent of $h$, and then demanding consistency with other parameters/variables describing the surface layer. We start by proposing that the material properties $\bar{\beta}=\bar{\beta}(z ; \boldsymbol{n}), \bar{\kappa}=\bar{\kappa}(z ; \boldsymbol{n}), \bar{\chi}(z)$, and $\bar{w}(z)$ are distributed along the $z$-direction according to

$$
\begin{aligned}
\bar{\beta} & =\frac{1}{h} D_{\beta}(z / h ; \boldsymbol{n}) \beta(\boldsymbol{n}), \\
\bar{\kappa} & =\frac{1}{h} D_{\kappa}(z / h ; \boldsymbol{n}) \kappa(\boldsymbol{n}), \\
\bar{\chi} & =\frac{1}{h} D_{\chi}(z / h) \chi, \\
\bar{w} & =\frac{1}{h} D_{w}(z / h) w,
\end{aligned}
$$

where $D_{\beta}, D_{\kappa}, D_{\chi}$, and $D_{w}$ satisfy the normalization conditions

$$
\frac{1}{h} \int_{0}^{h} \mathrm{~d} z D_{\beta}=1, \quad \frac{1}{h} \int_{0}^{h} \mathrm{~d} z D_{\kappa}=1, \quad \frac{1}{h} \int_{0}^{h} \mathrm{~d} z D_{\chi}=1, \quad \frac{1}{h} \int_{0}^{h} \mathrm{~d} z D_{w}=1 .
$$


As a result the integrated quantities

$$
\beta=\int_{0}^{h} \mathrm{~d} z \bar{\beta}, \quad \kappa=\int_{0}^{h} \mathrm{~d} z \bar{\kappa}, \quad \chi=\int_{0}^{h} \mathrm{~d} z \bar{\chi}, \quad w=\int_{0}^{h} \mathrm{~d} z \bar{w}
$$

are independent of $h$.

We further propose that the director scales as

$$
\boldsymbol{n}(z)=\boldsymbol{n}^{(0)}+h \boldsymbol{n}^{(1)}(z / h)+O\left(h^{2}\right),
$$

and the velocity scales as in equation (3.11):

$$
\boldsymbol{v}(z)=\boldsymbol{v}^{(0)}+h \boldsymbol{v}^{(1)}(z / h)+O\left(h^{2}\right),
$$

such that $\nabla \boldsymbol{n}$ and $\nabla \boldsymbol{v}$ both scale as $h^{0}$. Consequently, $\boldsymbol{\sigma}^{d}, \boldsymbol{\sigma}^{v}$, and $\boldsymbol{H}^{v}$, in which only the first-order derivatives $\nabla \boldsymbol{n}$ and $\nabla \boldsymbol{v}$ are involved, all scale as $h^{0}$ to the leading order, while $\boldsymbol{H}^{d}$ ( $=K \nabla^{2} \boldsymbol{n}$ under the one-constant approximation) scales as $\partial_{z}^{2} \boldsymbol{n} \sim h^{-1}$.

\subsection{Liquid-crystal boundary conditions}

It follows from the scaling relations that to the leading order $O\left(h^{-1}\right)$, the equation of tangential force balance in the surface layer can be written as

$$
-\partial_{i} p+\partial_{z} \sigma_{z i}^{d}+\partial_{z} \sigma_{z i}^{v}+g_{i}^{w}+g_{i}^{s}+g_{i}^{c}=0,
$$

for $i=x, y$ along the solid surface. Here we have used the fact that to the leading order, $\partial_{j} \sigma_{j i}^{d}$ can be approximated by $\partial_{z} \sigma_{z i}^{d}$ and scales as $\partial_{z}^{2} n \sim h^{-1}$. (Under the oneconstant approximation, $\partial_{j} \sigma_{j i}^{d}=-K \nabla^{2} n_{k} \partial_{i} n_{k}$ and $\partial_{z} \sigma_{z i}^{d}=-K \partial_{z}^{2} n_{k} \partial_{i} n_{k}-K \partial_{z} n_{k} \partial_{i} \partial_{z} n_{k}$, both equal to $-K \partial_{z}^{2} n_{k} \partial_{i} n_{k}$ at the leading order of $h^{-1}$.) Similarly, $\partial_{j} \sigma_{j i}^{v}$ is approximated by $\partial_{z} \sigma_{z i}^{v}$ and scales as $\partial_{z}^{2} v \sim h^{-1}$. It is also noted that the force densities $g_{i}^{w}, g_{i}^{s}$, and $g_{i}^{c}$ all scale as $h^{-1}$. Using the natural boundary condition (5.21) and the scaling of $\bar{\beta}$, $\bar{\kappa}, \bar{\chi}$, and $\bar{w}$, we integrate equation (5.29) along the $z$-direction from 0 to $h$ to obtain one of the two boundary conditions:

$$
\left[\hat{z} \cdot\left(\boldsymbol{\sigma}^{d}+\left.\boldsymbol{\sigma}^{v}\right|_{z=h}+\boldsymbol{G}^{w}+\boldsymbol{G}^{s}+\boldsymbol{G}^{c}\right] \cdot(\mathbf{1}-\hat{z} \hat{z})=0,\right.
$$

in which

$$
\boldsymbol{G}^{w}=\int_{0}^{h} \mathrm{~d} z \boldsymbol{g}^{w}, \quad \boldsymbol{G}^{s}=\int_{0}^{h} \mathrm{~d} z \boldsymbol{g}^{s}, \quad \boldsymbol{G}^{c}=\int_{0}^{h} \mathrm{~d} z \boldsymbol{g}^{c}
$$

are the three surface-layer-integrated forces. In deriving equation (5.30) from (5.29), we keep only those terms that scale as $h^{0}$ and hence the term $\int_{0}^{h} \mathrm{~d} z\left[-\partial_{i} p\right] \sim h$ drops out. In the $h \rightarrow 0^{+}$limit, $\left.\left.\boldsymbol{\sigma}^{d}\right|_{z=h} \rightarrow \boldsymbol{\sigma}^{d}\right|_{z=0^{+}},\left.\left.\boldsymbol{\sigma}^{v}\right|_{z=h} \rightarrow \boldsymbol{\sigma}^{v}\right|_{z=0^{+}}, \boldsymbol{n}^{(0)} \rightarrow \boldsymbol{n}$ and $\boldsymbol{v}^{(0)} \rightarrow \boldsymbol{v}$, and equation (5.30) becomes a hydrodynamic boundary condition that governs the tangential momentum transport across the fluid-solid interface $z=0^{+}$.

In equation (5.14) for the balance of molecular fields, $\boldsymbol{H}^{d}, \boldsymbol{H}^{w}, \boldsymbol{H}^{r}$, and $\boldsymbol{H}^{c}$ all scale as $h^{-1}$, while $\boldsymbol{H}^{v}$ scales as $h^{0}$. Therefore, integrating equation (5.14) along the $z$-direction from 0 to $h$ yields, to the leading order $O\left(h^{0}\right)$, another boundary condition:

$$
\boldsymbol{\Pi}^{d}+\boldsymbol{\Pi}^{w}+\boldsymbol{\Pi}^{r}+\boldsymbol{\Pi}^{c}-\Lambda \boldsymbol{n}=0,
$$

where

$$
\boldsymbol{\Pi}^{d}=\int_{0}^{h} \mathrm{~d} z \boldsymbol{H}^{d}, \quad \boldsymbol{\Pi}^{w}=\int_{0}^{h} \mathrm{~d} z \boldsymbol{H}^{w}, \quad \boldsymbol{\Pi}^{r}=\int_{0}^{h} \mathrm{~d} z \boldsymbol{H}^{r}, \quad \boldsymbol{\Pi}^{c}=\int_{0}^{h} \mathrm{~d} z \boldsymbol{H}^{c}
$$

are the surface molecular fields, with $\Lambda$ a Lagrange multiplier imposed by $\boldsymbol{n}^{2}=1$. In the $h \rightarrow 0^{+}$limit, $\left.\boldsymbol{\Pi}^{d} \rightarrow \hat{z} \cdot \pi\right|_{z=0^{+}}, \boldsymbol{n}^{(0)} \rightarrow \boldsymbol{n}, \boldsymbol{v}^{(0)} \rightarrow \boldsymbol{v}$, and equation (5.31) becomes 
the second hydrodynamic boundary condition that governs the molecular torque transport across the fluid-solid interface $z=0^{+}$.

To further express the boundary conditions in more physical terms, we note that according to equations (5.15), (5.16), and (5.17), the tangential part of $\boldsymbol{G}^{w}$ is given by

$$
\boldsymbol{G}^{w} \cdot(\mathbf{1}-\hat{z} \hat{z})=-\frac{w}{2} \nabla\left[\left(\boldsymbol{n}^{(0)} \cdot \hat{z}\right)^{2}\right] \cdot(\mathbf{1}-\hat{z} \hat{z}),
$$

$\boldsymbol{G}^{s}$ itself is tangential, given by

$$
\boldsymbol{G}^{s}=-\beta \boldsymbol{v}^{(0)} \cdot(\mathbf{1}-\hat{z} \hat{z})
$$

and $\boldsymbol{G}^{c}$ is also tangential, given by

$$
\boldsymbol{G}^{c}=\chi\left(\boldsymbol{n}^{(0)} \cdot \hat{z}\right) \dot{\boldsymbol{n}}^{(0)} \cdot(\mathbf{1}-\hat{z} \hat{z})
$$

(Note that $\boldsymbol{G}^{s}=\boldsymbol{G}^{s} \cdot(\mathbf{1}-\hat{z} \hat{z})$ and $\boldsymbol{G}^{c}=\boldsymbol{G}^{c} \cdot(\mathbf{1}-\hat{z} \hat{z})$ ) ) Also, from the definition $\boldsymbol{H}^{d}=\nabla \cdot \pi-\partial f_{d} / \partial \boldsymbol{n}$ and the natural boundary condition $\hat{z} \cdot \pi-\lambda_{0} \boldsymbol{n}=0$, we have $\boldsymbol{\Pi}^{d}=\int_{0}^{h} \mathrm{~d} z \boldsymbol{H}^{d}=\left.\hat{z} \cdot \pi\right|_{z=h}$, in which $\boldsymbol{H}^{d}$ is given by $H_{k}^{d}=\partial_{z} \pi_{z k} \sim h^{-1}$ to the leading order. The other three surface molecular fields may be expressed as $\boldsymbol{\Pi}^{w}=w\left(\boldsymbol{n}^{(0)} \cdot \hat{z}\right) \hat{z}$, $\boldsymbol{\Pi}^{r}=-\kappa \dot{\boldsymbol{n}}^{(0)}$, and $\boldsymbol{\Pi}^{c}=\chi\left(\boldsymbol{n}^{(0)} \cdot \hat{z}\right) \boldsymbol{v}^{(0)} \cdot(\mathbf{1}-\hat{z} \hat{z})$. Hence the two LC boundary conditions may be expressed as

$$
\begin{aligned}
& \left\{\hat{z} \cdot\left(\boldsymbol{\sigma}^{d}+\boldsymbol{\sigma}^{v}\right)-\frac{w}{2} \nabla\left[(\boldsymbol{n} \cdot \hat{z})^{2}\right]-\beta \boldsymbol{v}+\chi(\boldsymbol{n} \cdot \hat{z}) \dot{\boldsymbol{n}}\right\} \cdot(\mathbf{1}-\hat{z} \hat{z})=0, \\
& \hat{z} \cdot \pi+w(\boldsymbol{n} \cdot \hat{z}) \hat{z}-\kappa \dot{\boldsymbol{n}}+\chi(\boldsymbol{n} \cdot \hat{z}) \boldsymbol{v} \cdot(\mathbf{1}-\hat{z} \hat{z})-\Lambda \boldsymbol{n}=0 .
\end{aligned}
$$

Physically, equation (5.32) is a generalized slip boundary condition, describing the tangential force balance in the surface layer, where $\hat{z} \cdot \boldsymbol{\sigma}^{d} \cdot(\mathbf{1}-\hat{z} \hat{z})$ and $\hat{z} \cdot \boldsymbol{\sigma}^{v} \cdot(\mathbf{1}-\hat{z} \hat{z})$ are the stress components due to the elastic correlation and viscous coupling, respectively, $-\frac{1}{2} w \nabla\left[(\boldsymbol{n} \cdot \hat{z})^{2}\right] \cdot(\mathbf{1}-\hat{z} \hat{z})$ is the Marangoni force due to the variation of the surface alignment energy along the solid surface, $-\beta \boldsymbol{v} \cdot(\mathbf{1}-\hat{z} \hat{z})$ is the frictional force due to the slip relative to the solid surface, and $\chi(\boldsymbol{n} \cdot \hat{z}) \dot{\boldsymbol{n}} \cdot(\mathbf{1}-\hat{z} \hat{z})$ is the frictional force due to the director rotation. Similarly, equation (5.33) is a condition for weak anchoring, describing the balance of various molecular fields in the surface layer. Note that in using equations (5.32) and (5.33) as the hydrodynamic boundary conditions in the $h \rightarrow 0^{+}$limit, $\boldsymbol{v}=\boldsymbol{v} \cdot(\mathbf{1}-\hat{z} \hat{z})$ is tangent to the solid surface. The presence of the cross-coupling term in the boundary conditions can imply interesting consequences, e.g. flow-induced orientational switching in the weak anchoring limit.

\section{Concluding remarks}

Boundary conditions are sometimes considered within the context of kinetic theory, as in the case of rarefied gasdynamics. While kinetic theory has the advantage of being physically intuitive, it generally suffers from sensitivity to model details, such as interfacial geometries. Hence it is difficult to draw general conclusions from kinetic theory in regard to hydrodynamic boundary conditions. This is especially the case for dense liquids. In contrast, Onsager's variational principle takes the point of view of ensuring force balance arising from the dynamics of energetic and dissipative processes, so that the derived equations of motion, together with the boundary conditions, can lead to hydrodynamics that predicts the most probable course of motion. Hence the general approach presented here does not address the details of the interfacial model. Instead, only a phenomenological framework is established, in 
which the hydrodynamic boundary conditions constitute an inherent component; and within this framework the details of the interfacial model are swept into the relevant parameters. In this context our work represents only a beginning to the understanding of interfacial phenomena.

It should also be noted that the boundary conditions presented in this work are inherently in the linear response regime. This is inevitable since they arise from the Onsager principle. Deviations are thus expected, for example, in the high-shearrate regimes. The deviations may take two different forms. In the incipient stage of nonlinearity, it may be expected that only the parameters acquire a shear-rate dependence. For example, $\beta$ may no longer be a constant but can acquire a dependence on the magnitude of the slip velocity. However, deep in the nonlinear regime even the form of the boundary condition may become invalid. When that happens one has to re-examine the basic physics involved, and new models and frameworks will be required.

We would like to thank Congmin $\mathrm{Wu}$ for the molecular dynamics results shown in figure 2. Support of this work by RGC grant CA05/06.SC01 and No. 602007 is gratefully acknowledged.

\section{Appendix A. Molecular dynamics simulations of a confined simple fluid}

The MD simulations were performed for a simple fluid confined between two planar solid walls parallel to the $(x, y)$-plane. Interaction between the fluid molecules was modelled by the Lennard-Jones potential $U_{\mathrm{FF}}(r)=4 \epsilon\left[(\sigma / r)^{12}-(\sigma / r)^{6}\right]$, where $r$ is the distance between the molecules, $\epsilon$ and $\sigma$ are the energy and range parameters, respectively. Each of the two walls was constructed by four [001] planes of an fcc lattice, with each solid atom attached to the lattice site by a harmonic spring. The mean-squared displacement of solid atoms was controlled to obey the Lindemann criterion. The fluid-solid interaction was modelled by a modified Lennard-Jones potential $U_{\mathrm{FS}}(r)=4 \epsilon_{\mathrm{FS}}\left[\left(\sigma_{\mathrm{FS}} / r\right)^{12}-\delta\left(\sigma_{\mathrm{FS}} / r\right)^{6}\right]$, with energy and range parameters $\epsilon_{\mathrm{FS}}=1.16 \epsilon$ and $\sigma_{\mathrm{FS}}=1.04 \sigma$, and a parameter $\delta$ for changing the wetting property of the fluid. Both $U_{\mathrm{FF}}$ and $U_{\mathrm{FS}}$ were cut off at $r_{c}=2.5 \sigma$. The mass of the solid atom was set equal to that of the fluid molecule $m$, and the average number densities of the fluid and wall were set at $\rho=0.81 \sigma^{-3}$ and $\rho_{w}=1.86 \sigma^{-3}$, respectively. The temperature was controlled at $1.4 \epsilon / k_{B}$, where $k_{B}$ is Boltzmann's constant. The two walls were separated by a distance of $16 \sigma$ along the $z$-direction. Moving the top wall at a constant velocity $1.0(\epsilon / m)^{1 / 2}$ in the $x$-direction relative to the bottom wall induces Couette flow. Periodic boundary conditions were imposed in the $x$ - and $y$-directions. More technical details can be found in Qian et al. (2003).

\section{Appendix B. Interfacial stress components and wall potential}

Consider a fluid in the half-space $z \geqslant 0$ bounded by a smooth solid surface at $z=0$. Here we consider only the short-range component of the wall potential. Hence the fluid is in the wall potential $V_{w}(z)$ that has a finite force range, i.e. $V_{w}(z)=$ const. for $z \geqslant h$. Consistent with the illustration in figure 1 , the constant $V_{w}(z)$ beyond $z=h$ is set to be zero in what follows. The hydrostatic pressure distribution satisfies

$$
-\partial_{z} p-n \partial_{z} V_{w}=0,
$$


from which the pressure can be expressed as

$$
p(z)=p_{B}-\int_{h}^{z} \mathrm{~d} z^{\prime}\left[n\left(z^{\prime}\right) \partial_{z^{\prime}} V_{w}\left(z^{\prime}\right)\right],
$$

with $p_{B}$ denoting the constant pressure beyond $z=h$, guaranteed by the constancy of $V_{w}$ in the bulk. According to Kirkwood \& Buff (1949), the fluid-solid interfacial tension $\gamma_{\mathrm{FS}}$ is given by

$$
\gamma_{\mathrm{FS}}=\int_{0}^{\infty} \mathrm{d} z\left[\sigma_{\|}(z)-\sigma_{\perp}(z)\right]
$$

where $\sigma_{\|}$and $\sigma_{\perp}$ are the parallel and normal components of the stress, i.e. $\sigma_{\|}=\sigma_{x x}=\sigma_{y y}$ and $\sigma_{\perp}=\sigma_{z z}$. Note that in defining the stress components $\sigma_{\|}$and $\sigma_{\perp}$, fluid-fluid and fluid-solid interactions both need to be taken into account. Therefore, the hydrostatic equation of the force balance in the $z$-direction, equation (B 1), can be rewritten as $\partial_{z} \sigma_{\perp}=0=-\partial_{z} p-n \partial_{z} V_{w}$, with $\sigma_{\perp}(z)$ being constant in the $z$-direction, given by $-p_{B}$ in the bulk. Therefore, we have

$$
\sigma_{\perp}(z)=-p(z)-\int_{h}^{z} \mathrm{~d} z^{\prime}\left[n\left(z^{\prime}\right) \partial_{z^{\prime}} V_{w}\left(z^{\prime}\right)\right]
$$

according to equation (B 2). From equation (B 4) the difference between $\sigma_{\perp}(z)$ and $-p(z)$ within the surface layer is seen to be induced by the normal wall force density. But since the wall force density is in the $z$-direction (with the atomic-scale lateral inhomogeneities of the solid surface already smoothed out), the parallel component of the stress is simply given by $\sigma_{\|}(z)=-p(z)$. It follows from equation (B 3$)$ that the interfacial tension $\gamma_{\mathrm{FS}}$ can be written as

$$
\gamma_{\mathrm{FS}}=\int_{0}^{h} \mathrm{~d} z\left\{\int_{h}^{z} \mathrm{~d} z^{\prime}\left[n\left(z^{\prime}\right) \partial_{z^{\prime}} V_{w}\left(z^{\prime}\right)\right]\right\} .
$$

Introducing a quantity with the dimension of energy density $\bar{\gamma}_{\mathrm{FS}}$ through the relation $\gamma_{\mathrm{FS}}=\int_{0}^{\infty} \mathrm{d} z \bar{\gamma}_{\mathrm{FS}}(z)$, we have

$$
\bar{\gamma}_{\mathrm{FS}}(z)=\int_{h}^{z} \mathrm{~d} z^{\prime}\left[n\left(z^{\prime}\right) \partial_{z^{\prime}} V_{w}\left(z^{\prime}\right)\right]
$$

which vanishes beyond $z=h$. The fact that $\bar{\gamma}_{\mathrm{FS}}$ is finite only within a short distance of the solid wall is verified by MD simulation results in figure 2(b), and lends support to the concept that $\gamma_{\mathrm{FS}}$ is an interfacial quantity, as well as to the physical existence of a surface layer.

\section{Appendix C. Derivation of the reduced action functional $A^{\mathrm{SL}}$}

Here we present the details involved in deriving the reduced action functional $A^{\mathrm{SL}}$ in equation (3.14) for the surface layer. We start with $\Phi_{v}=\frac{1}{4} \int \mathrm{d} \boldsymbol{r}\left[\eta\left(\partial_{i} v_{j}+\partial_{j} v_{i}\right)^{2}\right]$ in which neither $\partial_{x} v_{k}\left(\cong \partial_{x} v_{k}^{(0)}\right)$ nor $\partial_{z} v_{k}\left(\cong \partial_{\zeta} v_{k}^{(1)}\right)$ depends on $h$ to the leading order. Consequently, the integral $\Phi_{v}=\frac{1}{4} \int \mathrm{d} S \int_{0}^{h} \mathrm{~d} z\left[\eta\left(\partial_{i} v_{j}+\partial_{j} v_{i}\right)^{2}\right]$ scales as $h$. That is, to the leading order the viscous dissipation in the surface layer contributes a surface integral of order $\sim \int \mathrm{d} S[h]$, and is thus negligible. 
For the next term $\Phi_{s}=\frac{1}{2} \int \mathrm{d} r\left[\bar{\beta} v_{x}^{2}\right]=\frac{1}{2} \int \mathrm{d} S \int_{0}^{h} \mathrm{~d} z\left[\bar{\beta}(z, \phi) v_{x}^{2}\right]$, we have $v_{x} \cong v_{x}^{(0)}$, $\beta(\phi)=\int_{0}^{h} \mathrm{~d} z[\bar{\beta}(z ; \phi)]$, and $\phi \cong \phi^{(0)}$, all independent of $h$ to the leading order. Thus

$$
\Phi_{s}=\frac{1}{2} \int \mathrm{d} S\left[\beta v_{x}^{2}\right]
$$

where $v_{x}\left(\cong v_{x}^{(0)}\right)$ depends on $x$ only, measuring the local slippage of the whole surface layer.

For $\Phi_{d}=\frac{1}{2} \int \mathrm{d} \boldsymbol{r}\left[J^{2} / M\right]$, we use the fact that in the surface layer, $J \cong J_{z} \hat{z}, J_{z} \cong-\dot{\phi} z$, and $\dot{\phi} \cong \partial \phi^{(0)} / \partial t+v_{x}^{(0)} \partial_{x} \phi^{(0)}$ is nearly constant, so that

$$
\Phi_{d}=\frac{1}{2} \int \mathrm{d} S \int_{0}^{h} \mathrm{~d} z\left[\frac{J_{z}^{2}}{M}\right]=\frac{1}{2} \int \mathrm{d} S \int_{0}^{h} \mathrm{~d} z\left[\frac{\dot{\phi}^{2} z^{2}}{M}\right]=\frac{1}{2} \int \mathrm{d} S\left[\frac{\dot{\phi}^{2}}{\Gamma}\right]
$$

where

$$
\frac{1}{\Gamma}=\int_{0}^{h} \mathrm{~d} z\left[\frac{z^{2}}{M}\right]=\int_{0}^{1} \mathrm{~d} \zeta\left[\frac{\zeta^{2}}{m}\right]
$$

In the last step on the right-hand side of equation (C 3), we have used (3.9) and the definition $\zeta=z / h$.

For $\dot{F}$, the routine is similar, i.e. from equations $(2.10)$ to $(2.12)$, we have

$$
\begin{aligned}
& \int \mathrm{d} S \int_{0}^{h} \mathrm{~d} z\left[\left(\mu_{\mathrm{CH}}+\frac{\partial \bar{\gamma}_{\mathrm{FS}}}{\partial \phi}\right) \frac{\partial \phi}{\partial t}\right] \\
& \cong \int \mathrm{d} S\left\{\left(-\frac{3 \gamma \xi}{2 \sqrt{2}} \int_{0}^{h} \mathrm{~d} z\left[\partial_{z}^{2} \phi\right]+\int_{0}^{h} \mathrm{~d} z\left[\frac{\partial \bar{\gamma}_{\mathrm{FS}}}{\partial \phi}\right]\right) \frac{\partial \phi}{\partial t}\right\} \\
& =\int \mathrm{d} S\left\{\left[-\frac{3 \gamma \xi}{2 \sqrt{2}}\left(\left.\partial_{z} \phi\right|_{z=h}\right)+\frac{\partial \gamma_{\mathrm{FS}}}{\partial \phi}\right] \frac{\partial \phi}{\partial t}\right\},
\end{aligned}
$$

in which we have used the relation $\mu_{\mathrm{CH}} \cong-3 \gamma \xi /(2 \sqrt{2}) \partial_{z}^{2} \phi \sim 1 / h$, and the natural boundary condition $\left.\partial_{z} \phi\right|_{z=0}=0$ (so that the surface integral of $L_{\mathrm{CH}}$ in equation (2.10) is zero).

By combining the above results, we obtain for the surface layer the reduced action functional $A^{\mathrm{SL}}$ in equation (3.14) to the leading order of $h^{0}$.

\section{Appendix D. Derivation of the hydrodynamic boundary condition of the Allen-Cahn type}

Here we present the details involved in deriving equation (3.24), which leads to the hydrodynamic boundary condition (3.18) at $z=0^{+}$, in the $h \rightarrow 0^{+}$limit.

In the surface layer, the leading-order contribution to $\dot{\phi}$ is $\partial \phi^{(0)} / \partial t+v_{x}^{(0)} \partial_{x} \phi^{(0)}$, which is on the order of $h^{0}$. Thus the leading-order contribution to the right-hand side should be the same order. Substituting the total chemical potential expression

$\mu=\mu_{\mathrm{CH}}+\frac{\partial \bar{\gamma}_{\mathrm{FS}}}{\partial \phi}=\frac{3 \gamma}{2 \sqrt{2}}\left[-\xi\left(\partial_{x}^{2} \phi+\partial_{z}^{2} \phi\right)+\frac{1}{\xi} \phi\left(\phi^{2}-1\right)\right]+\frac{1}{h} \frac{\partial}{\partial \phi}\left[D_{\gamma}\left(\frac{z}{h} ; \phi\right) \gamma_{\mathrm{FS}}(\phi)\right]$ 
and the mobility coefficient $M=h^{3} m(\zeta ; \phi)$ into $\nabla \cdot[M \nabla \mu]$ yields

$$
\begin{aligned}
\nabla \cdot[M \nabla \mu]=\nabla \cdot\left(h ^ { 3 } m ( \frac { z } { h } ; \phi ) \nabla \left\{\frac{3 \gamma}{2 \sqrt{2}}[\right.\right. & \left.-\xi\left(\partial_{x}^{2} \phi+\partial_{z}^{2} \phi\right)+\frac{1}{\xi} \phi\left(\phi^{2}-1\right)\right] \\
& \left.\left.+\frac{1}{h} \frac{\partial}{\partial \phi}\left[D_{\gamma}\left(\frac{z}{h} ; \phi\right) \gamma_{\mathrm{FS}}(\phi)\right]\right\}\right) .
\end{aligned}
$$

By retaining only the leading-order $\left(h^{0}\right)$ terms, we obtain

$$
\dot{\phi}=\partial_{\zeta}\left(m\left(\zeta ; \phi^{(0)}\right) \partial_{\zeta}\left\{-\frac{3 \gamma \xi}{2 \sqrt{2}} \partial_{\zeta}^{2} \phi^{(1)}+\left.\frac{\partial}{\partial \phi}\left[D_{\gamma}(\zeta ; \phi) \gamma_{\mathrm{FS}}(\phi)\right]\right|_{\phi=\phi^{(0)}}\right\}\right) .
$$

Introducing the function

$$
\mu^{(0)}\left(\zeta ; \phi^{(0)}\right)=-\frac{3 \gamma \xi}{2 \sqrt{2}} \partial_{\zeta}^{2} \phi^{(1)}+\left.\frac{\partial}{\partial \phi}\left[D_{\gamma}(\zeta ; \phi) \gamma_{\mathrm{FS}}(\phi)\right]\right|_{\phi=\phi^{(0)}}
$$

for the total chemical potential $\mu_{\mathrm{CH}}+\partial \bar{\gamma}_{\mathrm{FS}} / \partial \phi \cong(1 / h) \mu^{(0)}\left(\zeta ; \phi^{(0)}\right)$ to the leading order of $h^{-1}$, we can re-write equation (D2) as

$$
\dot{\phi}=\frac{\partial \phi^{(0)}}{\partial t}+v_{x}^{(0)} \partial_{x} \phi^{(0)}=\partial_{\zeta}\left\{m\left(\zeta ; \phi^{(0)}\right) \partial_{\zeta}\left[\mu^{(0)}\left(\zeta ; \phi^{(0)}\right)\right]\right\},
$$

from which we obtain

$$
\partial_{\zeta}\left[\mu^{(0)}\left(\zeta ; \phi^{(0)}\right)\right]=\frac{\dot{\phi} \zeta}{m\left(\zeta ; \phi^{(0)}\right)} .
$$

Here we have used the facts that $\dot{\phi}$ is independent of $\zeta$ and $\partial_{\zeta} \mu^{(0)}=0$ at $\zeta=0$. It follows that the dependence of $\mu^{(0)}\left(\zeta ; \phi^{(0)}\right)$ on $\zeta$ is in the form of

$$
\mu^{(0)}\left(\zeta ; \phi^{(0)}\right)=\dot{\phi} \int_{1}^{\zeta} \mathrm{d} \zeta^{\prime}\left[\frac{\zeta^{\prime}}{m\left(\zeta^{\prime} ; \phi^{(0)}\right)}\right]+\mu^{(0)}\left(1 ; \phi^{(0)}\right)
$$

From equation (3.13), it can be deduced that $\mu^{(0)}\left(\zeta ; \phi^{(0)}\right) \propto h^{0}$ within the surface layer in limit of $h \rightarrow 0^{+}$. Since the total chemical potential $\mu \sim h^{-1}$ (equation (3.13)) has to match the bulk value at $\zeta=1$, and the bulk value is a constant $\sim h^{0}$, it follows that $\mu\left(\zeta=1 ; \phi^{(0)}\right)=\mu^{(0)}\left(1 ; \phi^{(0)}\right) / h \sim h^{0}$, so that $\mu^{(0)}\left(1 ; \phi^{(0)}\right) \propto h$ and thus negligible as $h \rightarrow 0^{+}$. It follows that equation (D 5) may be written as

$$
\mu^{(0)}\left(\zeta ; \phi^{(0)}\right)=\dot{\phi} \int_{1}^{\zeta} \mathrm{d} \zeta^{\prime}\left[\frac{\zeta^{\prime}}{m\left(\zeta^{\prime} ; \phi^{(0)}\right)}\right]
$$

with $\mu^{(0)}\left(1 ; \phi^{(0)}\right)=0$, and hence the surface-layer-integrated total chemical potential is of the integral form

$$
\int_{0}^{h} \mathrm{~d} z\left[\mu_{\mathrm{CH}}+\frac{\partial \bar{\gamma}_{\mathrm{FS}}}{\partial \phi}\right]=\int_{0}^{1} \mathrm{~d} \zeta\left[\mu^{(0)}\left(\zeta ; \phi^{(0)}\right)\right]=\dot{\phi} \int_{0}^{1} \mathrm{~d} \zeta\left\{\int_{1}^{\zeta} \mathrm{d} \zeta^{\prime}\left[\frac{\zeta^{\prime}}{m\left(\zeta^{\prime} ; \phi^{(0)}\right)}\right]\right\} .
$$

In the above, $\int_{0}^{1} \mathrm{~d} \zeta\left[\mu^{(0)}\left(\zeta ; \phi^{(0)}\right]\right.$ can be evaluated as

$$
\begin{aligned}
& \int_{0}^{1} \mathrm{~d} \zeta\left\{-\frac{3 \gamma \xi}{2 \sqrt{2}} \partial_{\zeta}^{2} \phi^{(1)}+\left.\frac{\partial}{\partial \phi}\left[D_{\gamma}(\zeta ; \phi) \gamma_{\mathrm{FS}}(\phi)\right]\right|_{\phi=\phi^{(0)}}\right\} \\
& =-\left.\frac{3 \gamma \xi}{2 \sqrt{2}}\left[\partial_{\zeta} \phi^{(1)}\right]\right|_{\zeta=1}+\left.\frac{\partial \gamma_{\mathrm{FS}}(\phi)}{\partial \phi}\right|_{\phi=\phi^{(0)}},
\end{aligned}
$$


by using the boundary condition $\partial_{\zeta} \phi^{(1)}=0$ at $\zeta=0$ and the normalization condition $\int_{0}^{1} \mathrm{~d} \zeta\left[D_{\gamma}(\zeta ; \phi)\right]=1$. Hence equation (D 7) may be re-expressed as

$$
\dot{\phi}=-\Gamma\left\{-\left.\frac{3 \gamma \xi}{2 \sqrt{2}}\left[\partial_{\zeta} \phi^{(1)}\right]\right|_{\zeta=1}+\left.\frac{\partial \gamma_{\mathrm{FS}}(\phi)}{\partial \phi}\right|_{\phi=\phi^{(0)}}\right\},
$$

where

$$
\frac{1}{\Gamma}=-\int_{0}^{1} \mathrm{~d} \zeta\left\{\int_{1}^{\zeta} \mathrm{d} \zeta^{\prime}\left[\frac{\zeta^{\prime}}{m\left(\zeta^{\prime} ; \phi^{(0)}\right)}\right]\right\}
$$

\section{REFERENCES}

BARRAT, J.-L. \& BOCQUET, L. 1999 Influence of wetting properties on hydrodynamic boundary conditions at a fluid/solid interface. Faraday Discuss. 112, 119-128.

Batchelor, G. K. 1967 An Introduction to Fluid Dynamics. Cambridge University Press.

Bocquet, L. \& BarRat, J.-L. 2007 Flow boundary conditions from nano- to micro-scales. Soft Matter 3, 685-693.

Briant, A. J. \& Yeomans, J. M. 2004 Lattice Boltzmann simulations of contact line motion. II. Binary fluids. Phys. Rev. E 69, 031603.

BRINKMAN, H. C. 1947 A calculation of the viscous force exerted by a flowing fluid on a dense swarm of particles. Appl. Sci. Res. A1, 27-34.

CAHn, J. W. \& Hilliard, J. E. 1958 Free energy of a nonuniform system. I. Interfacial free energy. J. Chem. Phys. 28, 258-267.

Chen, H. Y., Jasnow, D. \& Vinals, J. 2000 Interface and contact line motion in a two phase fluid under shear flow. Phys. Rev. Lett. 85, 1686-1689.

Dussan V., E. B. 1976 The moving contact line: the slip boundary condition. J. Fluid Mech. 77, 665-684.

Dussan V., E. B. 1979 On the spreading of liquids on solid surfaces: static and dynamic contact lines. Annu. Rev. Fluid Mech. 11, 371-400.

Dussan V., E. B. \& Davis, S. H. 1974 On the motion of a fluid-fluid interface along a solid surface. J. Fluid Mech. 65, 71-95.

Ericksen, J. L. 1960 Anisotropic fluids. Arch. Rat. Mech. Anal. 4, 231-237.

Frank, F. C. 1958 On the theory of liquid crystals. Discuss. Faraday Soc. 25, 19-28.

De Gennes, P. G. 1985 Wetting: statics and dynamics. Rev. Mod. Phys. 57, 827-863.

De Gennes, P. G. \& Prost, J. 1993 The Physics of Liquid Crystals. Clarendon.

DE Groot, S. R. \& Mazur, P. 1984 Non-Equilibrium Thermodynamics. Dover.

Huh, C. \& Scriven, L. E. 1971 Hydrodynamic model of steady movement of a solid/liquid/fluid contact line. J. Colloid Interface Sci. 35, 85-101.

JACQMIN, D. 2000 Contact-line dynamics of a diffuse fluid interface. J. Fluid Mech. 402, 57-88.

Kirkwood, J. G. \& Buff, F. P. 1949 The statistical mechanical theory of surface tension. J. Chem. Phys. 17, 338-343.

Koplik, J., Banavar, J. R. \& Willemsen, J. F. 1988 Molecular dynamics of Poiseuille flow and moving contact lines. Phys. Rev. Lett. 60, 1282-1285.

LeSLIE, F. M. 1968 Some constitutive equations for liquid crystals. Arch Rat. Mech. Anal. 28, 265-283.

Moffatt, H. K. 1964 Viscous and resistive eddies near a sharp corner. J. Fluid Mech. 18, 1-18.

NAvier, C. L. M. H. 1823 Memoire sur les lois du movement des fluides. Mem. l'Acad. R. Sci. l'Inst. France 6, 389-440.

Onsager, L. $1931 a$ Reciprocal relations in irreversible processes. I. Phys. Rev. 37, 405-426.

OnSAGER, L. $1931 b$ Reciprocal relations in irreversible processes. II. Phys. Rev. 38, 2265-2279.

Oseen, C. W. 1933 The theory of liquid crystals. Trans. Faraday Soc. 29, 883-889.

Parodi, O. J. 1970 Stress tensor for a nematic liquid crystal. J. Phys. (Paris) 31, 581-584.

Pismen, L. M. \& Pomeau, Y. 2000 Disjoining potential and spreading of thin liquid layers in the diffuse-interface model coupled to hydrodynamics. Phys. Rev. E 62, 2480-2492.

Qian, T. Z., Wang, X. P. \& Sheng, P. 2003 Molecular scale contact line hydrodynamics of immiscible flows. Phys. Rev. E 68, 016306. 
Qian, T. Z., Wang, X. P. \& Sheng, P. 2004 Power-law slip profile of the moving contact line in two-phase immiscible flows. Phys. Rev. Lett. 93, 094501.

Qian, T. Z., WANG, X. P. \& ShEng, P. 2006 A variational approach to moving contact line hydrodynamics. J. Fluid Mech. 564, 333-360.

Rapini, A. \& Papoular, M. 1969 Distorsion d'une lamelle nematique sous champ magnetique, conditions d'ancrage aux parois. J. Phys. (Paris) Colloq. 30, C4-54-C4-56.

RAYleigh, LORD 1873 Some general theorems relating to vibrations. Proc. Math. Soc. Lond. 4, 357-368.

Seppecher, P. 1996 Moving contact lines in the Cahn-Hilliard theory. Intl J. Engng Sci. 34, 977-992.

Thompson, P. A. \& Robbins, M. O. 1989 Simulations of contact-line motion: slip and the dynamic contact angle. Phys. Rev. Lett. 63, 766-769.

Yue, P. T., Feng, J. J., Liu, C. \& Shen, J. 2004 A diffuse-interface method for simulating two-phase flows of complex fluids. J. Fluid Mech. 515, 293-317.

Zocher, H. 1933 The effect of a magnetic field on the nematic state. Trans. Faraday Soc. 29, 945-957. 